\title{
Las garrapatas de la República de Colombia
}

\author{
Ernesto Osorno Mesa
}

Rev. Acad. Colomb. Cienc. Ex. Fis. Nat. 1940, 4 (13): 6-24.

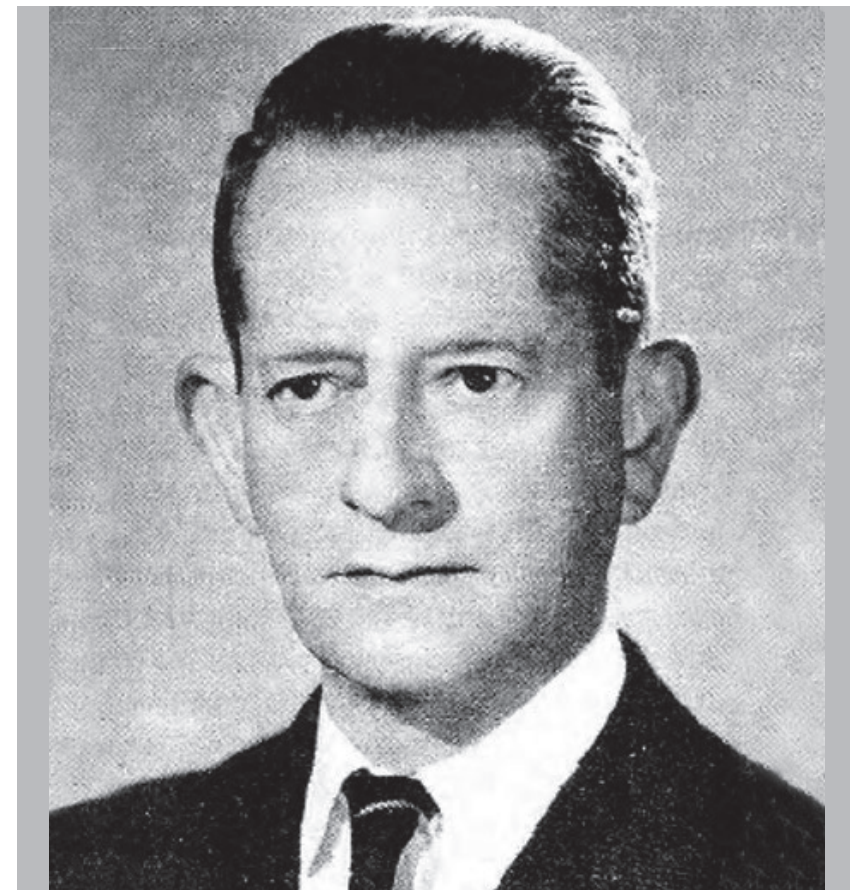

\section{Ernesto Osorno Mesa (1904-1976)}

Osorno Mesa es considerado como el fundador de los estudios de entomología médica en Colombia, ocupó la Silla No. 20 en la Academia Colombiana de Ciencias. Médico de la Universidad Nacional de Colombia (1927) y Doctor en Medicina y Cirugía (1935).

Fue nombrado médico jefe de las comisiones sanitarias antianémicas en los departamentos del Valle, Caldas y Cundinamarca, médico entomólogo en la sección de estudios especiales del Ministerio de Trabajo, Higiene y Previsión Social, médico jefe del laboratorio de entomología de la División de Malariología del Servicio Cooperativo Interamericano de Salud Pública (1947) y en 1964, fue designado como jefe del subgrupo de entomología en el Instituto Nacional de Salud. Sus investigaciones estuvieron encaminadas hacia artrópodos de interés médico. Se desempeñó como profesor de la cátedra de entomología de la Pontificia Universidad Javeriana (19441945) y de la cátedra de Biología y Parasitología de la Facultad de Medicina de la Universidad Nacional de Colombia (19511963). Fue autor de 51 publicaciones.
El trabajo académico seleccionado se puede considerar como un tesoro. Es un trabajo que requiere la atención de todos aquellos investigadores que han trabajado o trabajarán en este grupo de artrópodos.

Con la exposición de esta obra el doctor Osorno Mesa dio ingreso a la Academia Nacional de Medicina y recibió, en esa oportunidad, los elogios del doctor Roberto Franco quien manifestó que la comunicación "representa una de las contribuciones más valiosas a la parasitología de nuestra patria. A todos nuestros médicos y a todos los que se interesen por estos estudios debo recomendar encarecidamente este trabajo como el más completo que hasta hoy existe entre nosotros, con la convicción de que encontrarán en él la más segura y acertada guía para sus investigaciones".

Las garrapatas en general al ser transmisoras de enfermedades a los animales y a los humanos ocupan un lugar tan importante como el de los insectos y al ser hematófagas desempeñan un papel relevante en nuestro país.

En el estudio, inicialmente se detallan los caracteres generales de las garrapatas y posteriormente se presentan las claves para las familias Argasidae e Ixodidae del suborden Ixodoidea del orden Acari y sus respectivos géneros y especies incluyendo las descritas y halladas en Colombia y las conocidas de la República de Panamá.

Los hallazgos y las observaciones se obtuvieron con un trabajo de campo extenuante realizado en 15 departamentos de Colombia con los auspicios del Ministerio de Trabajo, Higiene y Previsión Social en cooperación con la División Sanitaria Internacional de la Fundación Rockefeller.

Paulina Muñoz, Magister

Miembro de Número 


\title{
LAS GARRAPATAS DE LA REPUBLICA DE COLOMBIA
}

\author{
ERNESTO OSORNO-MESA \\ Médico entomólogo de la Sección de Estudios Especiales \\ del Ministerio de Trabajo, Higiene y Previsión Social
}

EI presente estudio comprende las especies de garrapatas colombianas conocidas, como también las de la República de Panamá. En las claves incluyo las especies descritas y halladas en Colombia. (*).

Las referencias fueron tomadas de las descripciones originales y de todas las publicaciones sobre garrapatas de los dos países.

Colecté parte del material con el Dr. J. C. Bequaert, y parte, la obtuve por intermedio de la Fundación Rockefeller, en colaboración del Servicio de la Sección de Estudios Especiales del Ministerio de Trabajo, Higiene y Previsión Social.

Expreso mis agradecimientos a mi maestro, Dr. J. C. Bequaert, de la Universidad de Harvard, por la invaluable ayuda que gentilmente me prestó en la ejecución de este trabajo, y doy las gracias, igualmente, a los doctores Kerr, Boshell y Roca, por la aportación de ejemplares.

Caracteres generales de las garrapatas

Con el objeto de aclarar los términos técnicos usados en el presente estudio, doy a continuación una explicación general, por existir pocos vocablos castellanos aplicables a estos artrópodos. (Figs. 1-2).

El cuerpo está cubierto con una piel fuerte y coriácea, que se distiende enormemente, en ambos sexos de Argasidæ y en la hembra de Ixodidæ, por la sangre succionada al huésped. En ayuno, las garrapatas son aplanadas, de forma más o menos triangular en diseño, con patas prominentes y delgadas y un rostro en forma de pico en la parte anterior.

Las hembras en estado de repleción, simulan un fríjol o una gran semilla y los detalles anotados son difíciles de observar. En la mayor parte de las especies existe una rodela córnea situada en la parte anterior del dorso y conocida con el nombre de escudo. En el macho, el escudo cubre la mayor parte del dorso, mientras que en la hembra, unicamente una pequeña zona de la región anterior. Articulado con el margen anterior del escudo $\mathbf{y}$ generalmente dentro de una ligera muesca, hay una pieza pequeña, subtriangular, llamada capítulo o cabeza. El capítulo sostiene los palpos, las mandíbulas, los es. tuches mandibulares y el hipostoma. Los tres últimos órganos reunidos forman la proboscis o haustellum. El hipostoma es una pieza inferior y mediana (en realidad está formado por dos piezas), con numerosos dientes curvos, o dentecillos. Los dentecillos más cercanos a la base, forman filas, cuyo número ha sido usado para la diferenciación de las especies, aun cuando esto es variable. En la extre-

(*) Los estudios y observaciones en que se basa este trabajo se llevaron a cabo bajo los auspiclos del Ministerio de Trabajo, Higiene y Prevision Social en cooperacion con la División Sani. taria Internacional de la Fundacion Rockefeller. midad de las mandíbulas hay dos o tres protuberancias, conocidas con el nombre de apófosis, las cuales también han sido usadas en clasificación específica, pero ahora se sabe que este detalle es inconstante. El hipostoma y las mandíbulas penetran dentro de los tejidos del huésped cuando la garrapata se alimenta; adhiriéndose tan fuertemente, por medio de los dientes del hipostoma, que cuando se trata de separarla a viva fuerza, el cuerpo puede desarticularse del capítulo.

Los palpos se insertan a los lados de las piezas bucales. Están compuestos de cuatro segmentos, de los cuales se aprecian generalmente dos, porque el basal es corto $y$ ancho $y$ el apical es muy pequeño y a menudo situado en una depresión cerca de la extremidad del tercero. Los palpos son por lo regular cóncavos en la cara interna y pueden ocultar las piezas bucales. La longitud relativa del segundo y tercer segmentos de los palpos, suministra caracteres útiles para separar los géneros en las garrapatas. En la cara dorsal del capítulo en la hembra adulta hay dos áreas deprimidas, conocidas con el nombre de áreas porosas. Todavía nadie ha determinado su función. Toda hembra de la familia Ixodidce que no tenga estas áreas completamente desarrolladas, no está en estado adulto y no debe describirse como una especie nueva. La descripción de varias especies y aun géneros se ha basado en formas jovenes, debido a un descuido en la observación del detalle mencionado. Los géneros Phaulixodes, Herpetobia y Sarconyssus, están en este caso. El escudo es, por lo común, de forma irregularmente exagonal. En cada margen lateral hay una mancha pálida en forma de ojo; en algunos géneros los ojos faltan. El margen posterior del cuerpo, en la mayor parte de las especies, está limitado por un número de estrías cortas (8 a 10), las cuales diseñan una serie de 16 bulos o festones. Los festones son más marcados en el macho que en la hembra, en la cual son escasamente visibles cuando está distendida por la sangre. En la cara inferior del cuerpo o vientre hay dos aberturas medianas: la anterior situada cerca del capítulo, es el poro genital; la posterior es el ano. En muchas especies, detrás del ano, hay un surco curvo, de donde parte una estría mediana; en otras, el ano está contorneado hacia adelante por el surco, cuyas extremidades se dirigen hacia atrás, para terminar en el margen del cuerpo. En todas las formas hay un surco lateral a cada lado, que, dirigiéndose hacia adelante, llega al poro genital. En los machos de varios géneros hay una o dos placas triangulares, córneas, situadas a cada lado del ano, conocidas con el nombre de placas anales. Oca- 
sionalmente el abdomen termina en una protuberancia mediana, o una cola.

La larva, forma más joven nacida del huevo, tiene tres pares de patas. En el próximo estado de ninfa y en la hembra y macho del estado adulto posee cuatro pares. Las patas nacen de cada lado, en la parte anterior del vientre; las coxas están algunas veces muy juntas y otras veces separadas. Las patas son generalmente delgadas, de longitud casi igual, aunque el cuarto par es más largo y más grande que los demás. Cada una está compuesta por lo menos de seis segmentos que, de la base hacia la punta, están en el orden siguiente: coxa, trocánter, fémur, tibia, metatarso y tarso. El último, comúnmente está dividido en dos partes. En la extremidad del tarso hay un par de uñas grandes, situadas en un pedículo largo, entre las cuales hay generalmente un pulvillus. En la cara superior del tarso I existe un hoyuelo cubierto por una membrana $y$ conocido con el nombre de $6 r$ gano de Haller, su descubridor. Se supone que sea un brgano auditivo. Una o varias coxas poseen espinas, espolones, protuberancias o dientes. En los machos de algunas especies, la coxa posterior es enormemente ensanchada. Encima y ligeramente hacia atrás de la coxa posterior se encuentran las placas de los estigmas donde se abre el orificio del estigma o espiráculo. Cada placa es una pieza córnea, en cuya superficie hay granulaciones grandes y pequeñas. La forma de la placa del estigma es constante en cada especie, pero diferente en los distintos sexos.

En algunos géneros hay en el dorso, cerca de la mitad, un par de placas pequeñas, circulares $\mathrm{u}$ ovales, llamadas por el $\mathrm{Dr}$. Stiles placas porosas dorso-sub. medianas; todavía nadie ha determinado su importancia taxonómica.

Las garrapatas tienen, por lo geueral, una coloración uniforme; sin embargo, algunas for-

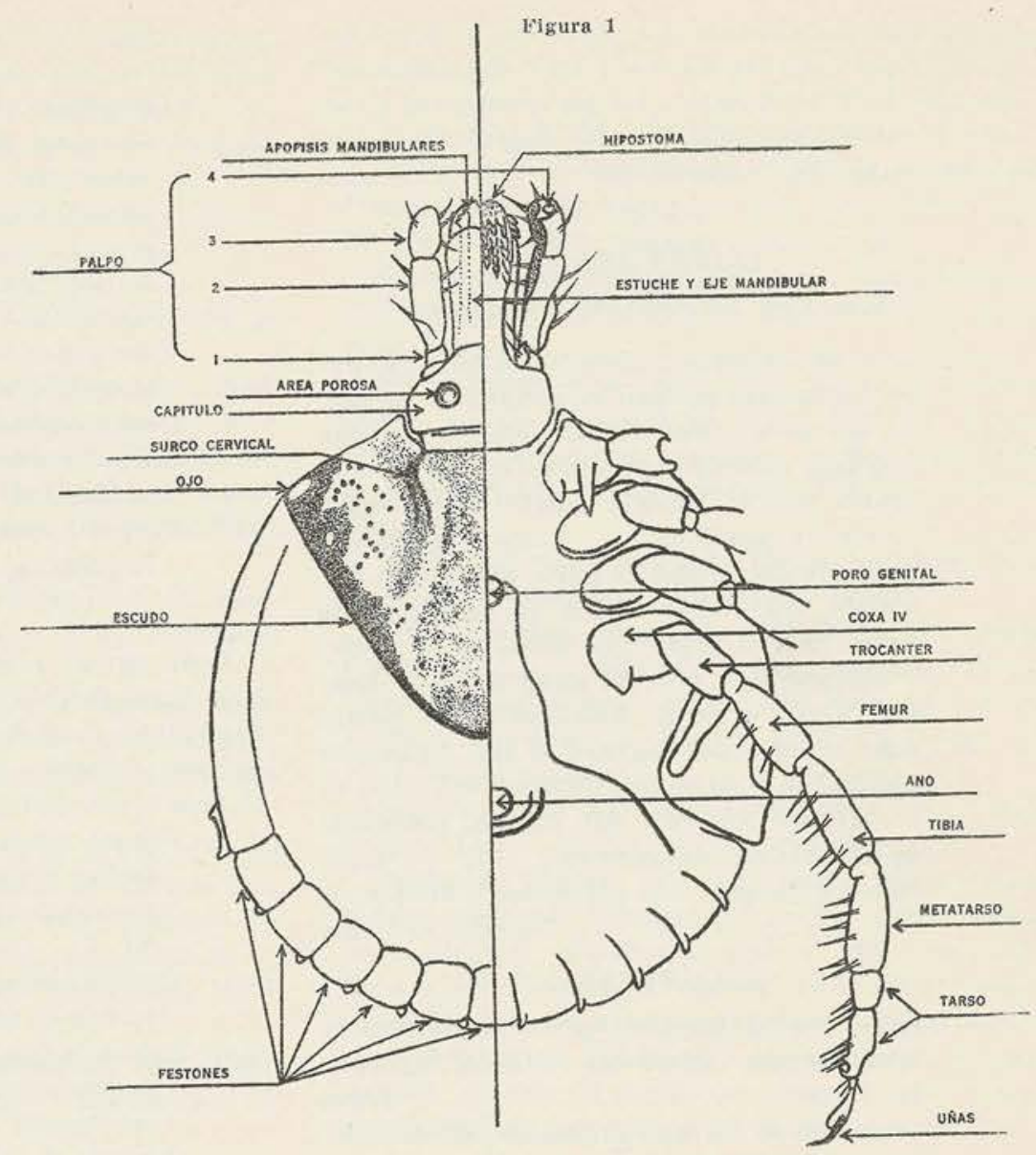

CARA DORSAL $Y$ VENTRAL DE LA HEMBRA DE AMBLYOMMA CAJENMENSE. (Aum. $21 \times$ )

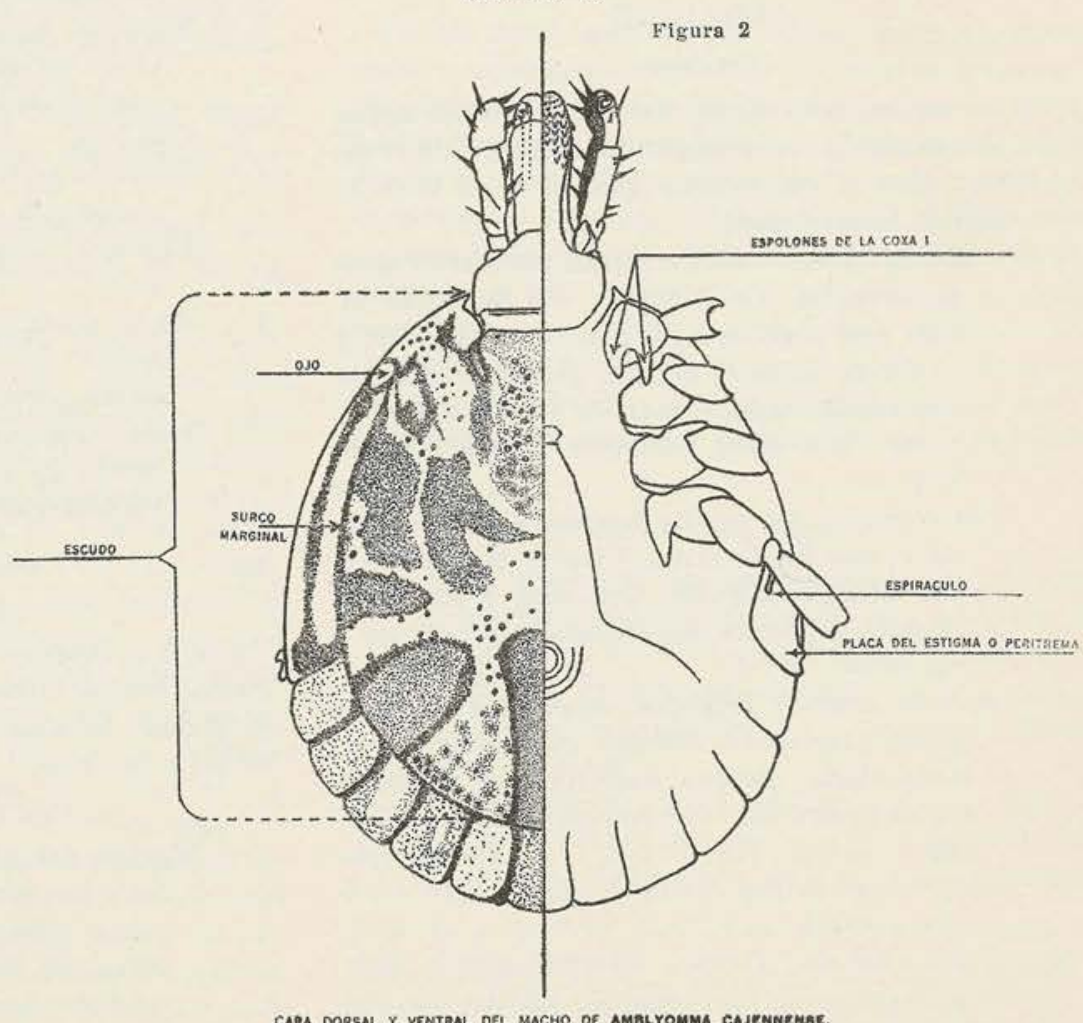

(A 1 m. $28 \times)$ 
mas poseen manchas de color castaño, blanco, amarillo o rojo. Cada especie tiene un color caracterís. tico, de valor taxonómico. En los ejemplares vivos, los colores se destacan mejor, que después de sumergirlos en líquidos preservativos.

\section{IXODOIDEA}

Clave para las Familias y Géneros

1 - Cuerpo semejante en ambos sexos, cubierto por un tegumento extensible, coriáceo y arrugado, sin escudo duro diferenciado en el dorso. Capítulo colocado ventralmente y a menudo oculto por el margen frontal del cuerpo. Coxas sin espolones .... Familia Argasidae

Tegumento del cuerpo en parte endurecido formando una rodela rígida (o escudo), que cubre toda la superficie dorsal en el macho y solamente la porción anterior en la hembra, ninfa y larva; únicamente las partes más blandas del tegumento son finamente punteadas o estriadas. Capítulo colocado en el extremo anterior del cuerpo, completamente visible dorsalmente. Una o varias coxas a menudo con espolones o aristas... ................. Familia Ixodidae

ARGASIDAE
1 - Cuerpo con el margen aplanado, delgado y
cortante, que difiere del resto del tegumen-
to $\ldots \ldots \ldots \ldots \ldots \ldots \ldots \ldots \ldots \ldots \ldots \ldots$.......................
Cuerpo con el margen grueso no definido, si-
milar al resto del tegumento ... Ornithodoros

IXODIDAE

\section{MACHOS}

Ocho patas; cara dorsal del cuerpo cubierta por una rodela rígida; cara dorsal del capítulo sin áreas porosas; vientre con orificio genital en el área intercoxal anteromediana.

1 - Vientre cubierto más o menos por siete placas endurecidas, no salientes, con un surco nítido que contornea el ano, frontalmente, y se dirige hacia el margen posterior. Escudo desornamentado, el margen posterior sin festones. Sin ojos; rostro y palpos alarga-

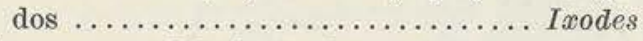

Surco curvo del vientre borroso o contorneando el ano hacia atrás. Vientre sin placas 0 con placas salientes. Con ojos o sin ellos. Margen posterior del escudo con festones 0 $\sin$ ellos $\ldots \ldots \ldots \ldots \ldots \ldots \ldots \ldots \ldots 2$

2 - Palpos largos y delgados, especialmente el segundo segmento. Escudo generalmente ornamentado, margen posterior con festones. Ojos presentes. Vientre sin placas salientes...$\ldots \ldots \ldots \ldots \ldots \ldots$ Amblyomma

Capítulo y palpos cortos y anchos, más o menos cónicos .................... 3

3 - Ojos ausentes. Escudo desornamentado, margen posterior con festones. Vientre sin placas salientes. Angulo externo del segundo segmento de los palpos más o menos salientes ................. Haemaphysatis Ojos presentes $\ldots \ldots \ldots \ldots \ldots \ldots \ldots \ldots \ldots 4$

4 - Vientre sin placas salientes. Coxas (especialmente las del cuarto par) a menudo muy anchas. Capítulo rectangular. Escudo por lo general ornamentado (excepto D. nitens), margen posterior con festones. Dermacentor

Vientre con cuatro placas posteriores, cuyos márgenes sobresalen hacia atrás. Coxas no ensanchadas. Capítulo exagonal. Escudo desornamentado .................. 5

5 - Margen posterior del escudo sin festones. Surco postanal borroso .......... Boophilus

Margen posterior del escudo con festones. Surco postanal nítido ......... Rhipicephatus

\section{HEMBRAS}

Ocho patas; cara dorsal del cuerpo dividida en una parte anterior dura y otra posterior blanda y extensible. Cara dorsal del capítulo con dos áreas porosas; vientre con un orificio genital (vulva) eu el área intercoxal anteromediana.

1 - Vientre con un surco curvo y nítido, que contornea el ano frontalmente. Sin ojos. Rostro y palpos alargados. Escudo desornamentado .................... Ixodes

Vientre con un surco curvo borroso, o que contornea el ano hacia atrás ............ 2

2 - Palpos largos y delgados, especialmente el segundo segmento. Ojos presentes. Escudo generalmente ornamentado ..... Amblyomma

Capítulo y palpos cortos y anchos más o menos cónicos .................... 3

3 - Sin ojos. Angulo externo del segundo segmento de los palpos más o menos salientes. Escudo desormamentado ...... Haemaphysalis

Con $\operatorname{ojos} \ldots \ldots \ldots \ldots \ldots \ldots \ldots \ldots \ldots \ldots . \ldots \ldots$

4 - Capítulo rectangular. Escudo generalmente ornamentado .............. Dermacentor

Capítulo exagonal. Escudo desornamenta-

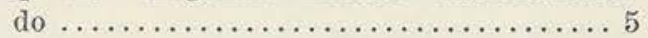

5 - Surco postanal borroso. Segundo y tercer segmento de los palpos muy corto, angulado en su parte externa .......... Boophilus

Surco postanal nítido. Segundo y tercer segmento de los palpos no tan corto, sin ángulos laterales salientes ....... Rhipicephalus

\section{Familia ARGASIDAE}

\section{$A R G A S$ Latreille}

Las dos especies de este género halladas en Colombia fueron introducidas indudablemente del Viejo Mundo. Se sabe únicamente que atacan aves de corral y palomas.

\section{Clave para las Especies}

1 - Margen del cuerpo deprimido, granujoso, dividido por una red de líneas, en placas numerosas, pequeñas, cuadrangulares o rectangulares. Espiráculo en media luna, mucho más pequeño que el anillo anal $\ldots \ldots \ldots \ldots \ldots$. ................... persicus (Oken) 
Margen del cuerpo deprimido, irregularmente arrugado, con numerosas estrías transver sales y generalmente algo vuelto hacia arriba. Espiráculo en media luna, aproximadamente del tamaño del anillo anal ........ ............... columbarum (Shaw)

\section{Argas persicus (Oken)}

Rhynchoprion persicum Oken, 1818, Isis, III, p. 1568, Pl. XIX, figs. 1-4 (Persia).

Argas persicus Neumann, 1896, Mém. Soc. Zool. France, IX, p. 7, figs. 4-5 ( $q$ ô, ninfa y larva). Nuttall, Warburton, Cooper and Robinson, 1908, Ticks, I, Argasidæ, p. 8, figs. 3-26, Pl. I, Fig. 3 (우 후, ninfa y larva). Dunn, 1929, Amer. Jl. Trop. Med., IX, p. 506 .

Argas persicus var. porteri Lehille, 1915, An. Zool. Aplicada, Santiago de Chile, II, p. 5, Pl. (San Bernardo, Prov. Santiago, Chile).

"? Argas chinche" "Goudot", in Gervais, 1844, Hist. Nat. Ins. Aptères, III, p. 462 (ataca al hombre en las tierras templadas de Colombia; no está deserita).

Argas americana Packard, 1873, Sixth Annual Rept. U. S. Geol. Survey Territories for 1872 , p. 740 , fig. 68 (Texas; sexo no especificado; "hallado en un lote de Ixodes bovis procedente de ganado").

Argas sanchezi Dugès, 1891, La Naturaleza, México, (2) I, p. 20, Pl. I.

Argas americanus firmatus Neumann, 1896, Mém. Soc. Zool. France, IX, p. 12 ( $q$; Constantine, Algeria).

Argas miniatus Koch, 1844, Arch.-f. Naturgesch., X, pt. 1, p. 219 (sexo no especificado; Demerara, British Guiana) ; 1847, Uebersicht d. Arachnidensyst., IV, pp. 12 and 32 , Pl. I. fig. 4.

Argas radiatus Railliet, 1893, Traité Zool. Médic. p. 718 (nombre nuevo para Argas americanus Packard, 1873).

Argas mauritianus Guérin-Méneville, 1844. Iconographie Règne Animal, Arachnides, p. 17, Pl. VI. figs. $3-3^{\text {a }}$ (Mauritius).

Colombia.-Todavía no he visto ejemplares procedentes de Colombia. Dunn (1929) lo anota como procedente de Barranquilla, en donde según él, esta garrapata abunda en los gallineros. Brumpt lo anota como procedente de Santa Marta, Magdalena (Alt. 4 mts., Temp. med. $30^{\circ} \mathrm{C}$.). (Col. N. Güete). Informe al Ministerio de T., H. y P. S., Oct. 1939). Neumann (1896) supuso que el "Argas chinche" de Goudot, era A. persious (=americanus) ; muy probablemente Goudot observó Ornithodoros rudis Karsch (=venezuelensis Brumpt), que es la garrapata común que ataca al hombre en las regiones templadas de Colombia. Además, Gervais (1844, p. 463) también anota: "Un Argas de las gallinas que M. Goudot colectó en una región cálida de Colombia. Es más grande que éste (chinche) y sin duda de especie diferente; obliga algunas veces a los propietarios a cambiar el gallinero". Estas ultimas observaciones se refieren claramente a Argas, de modo que Goudot aparentemente observó ambos, Argas y
Ornithodoros. De acuerdo con Dunn, es muy común en Panamá.

\section{Argas cohumbarum (Shaw)}

Acarus columbarum Shaw, 1793, in Shaw and Nodder, Vivarium Nature or the Natur. Misc., IV, Pl. CXXVIII (en palomas; Inglaterra).

Rhynchoprion columbae Hermann, 1804, Mémoire Aptérologique, p. 69, Pl. IV, figs. 10-11 (en palo. mas; Francia).

Argas marginatus Oken, 1815, Lehrb. d. Naturgesch., III, pt. 1, p. 402, Pl. XII, col. 5, fig. 3 (basado en Rhynchoprion columbae de Hermann). No es Acarus marginatus de Sulzer, 1776, ni de Fabricius, 1794.

Acarus reflexus Fabricius, 1794, Entom. Syst., IV, p. 426 ( $\sin$ especificar el huésped; Italia).

Argas reflexus Neumann, 1896, Mém. Soc. Zool. France, IX, p. 4, figs. $1-3$ ( $\hat{\imath} \hat{o}$; ninfa y larva). Nuttall, Warburton, Cooper and Robinson, 1908, Ticks, I, Argasidae, p. 22, figs. 27-33, Pl. I, figs. 1-2 (.$\hat{\delta}$; ninfa y larva).

Argas magnus Neumann, 1896, Mém. Soc. Zool. France, IX, p. 14, fig. 15 ( $\%$; Eeuador).

Colombia: Bogotá (Dr. R. Franco, 1905). Bogotá, en gallineros (Hernando Osorno, 1936). Sogamoso, Boyacá (Patiño C., Enero, 1939). Samacá, Boyacá (E. Brumpt, E. Osorno, Julio 1939). Sogamoso, Boyacá (E. Osorno, Octuhre 3, 1939). Sogamoso, Boyacá (E. Brumpt, E. Osorno, 1939). Tunja, Boyacá (Carlos Cárdenas G., 1940). Bogotá (Hernando Osorno, Septiembre 17, 1940).

\section{ORNITHODOROS Koch}

De las nueve especies que figuran en la siguiente clave, únicamente dos (talaje y rudis) se han anotado como procedentes de Colombia; tres (dunni, brodyi y azteci) se describieron de ejemplares de $\mathrm{Pa}$ namá. De las especies restantes, 0 . turicatus y $O$. coriaceus se sabe con certeza que son procedentes del sur de los Estados Unidos y de México; la procedencia de Sur América para estas dos especies es muy dudosa; O. furcosus se describió del Ecuador; o. megnini, "garrapata espinosa del oído" de los caballos y del ganado (accidentalmente del hombre), es común en el sur de los Estados Unidos y en México $\left(^{*}\right)$.

\section{Clave para las Especies}

1 - Ojos presentes, dos en cada lado, debajo del margen, distanciados entre sí y opuestos a las coxas I y II. Tegumento toscamente granujoso, excepto en algunas áreas grandes que son reticuladas.... 0 . coriaceus Koch

Ojos ausentes $\ldots \ldots \ldots \ldots \ldots \ldots \ldots \ldots \ldots 2$

2 - Tegumento ni con protuberancias, ni toscamente granujoso, punteado con hoyitos circulares, pequeños y superficiales. Extremi-

(*) La consideración sobre las aberraclones de los habitos y sobre la parte trasera de la armadura del hipostoma del adulto en o. megnint, 10 ha colocado algunas veces en un género aparte, el genero Otobius. Las especles con las alas movibles del camerostoma han sido separadas en el género Alectorobius Pocock, 1907 (tipo: Argas talaje Guerin). 
dad anterior sin forma cónica. Hipostoma muy corto redondeado en la punta, desarmado. Tegumento de la ninfa cubierto con muchas espinas o pelos erizados ......... ............... megnini (Dugès)

Tegumento con protuberancias o toscamente granujoso. Extremidad anterior de forma cónica. Hipostoma largo, dentado (algunas veces con dientes diminutos) .......... 3

3 - Dorso del tegumento con áreas grandes, notorias (o discos); sin protuberancias gruesas y voluminosas. Capítulo colocado en un hoyo profundo (camerostoma), con prolongamientos laterales en forma de alas prominentes y bordes movibles ................ 4

Dorso del tegumento con protuberancias uniformes, sin discos notorios . .......... 6

4 - Tegumento cubierto con protuberancias muy grandes, levantadas a manera de conos, especialmente a lo largo del margen posterior .............. o. talaje (Guérin)

Tegumento cubierto con protuberancias de tamaño pequeño o mediano que no se levantan a manera de conos ................ 5

5 - Pequeño (longitud del adulto $4 \mathrm{~mm}$. o menos), cubierto con protuberancias pequeñas, con muchos discos colocados como en forma de radios. Tarsos I y IV sin jorobas preapicales notorias. Parasita Murciélagos ........ ................ O. dunni Matheson

Más grande (la longitud del adulto pasa de $4 \mathrm{~mm}$.), cubierto con protuberancias de tamaño mediano, con pocos discos. Tarso I con joroba preapical; tarso IV sin jorobas. No parasita Murciélagos ...... O. rudis Karsch

6 - Capítulo colocado en un camerostoma con alas laterales prominentes y bordes movibles. . 7

Capítulo colocado en un camerostoma sin alas laterales movibles .............. 8

7 - Tarsos I y IV con una joroba preapical. Dorso gradualmente estrecho de atrás hacia el ápex anterior que es cónico. Hipostoma con dentecillos diminutos ...O. azteci Matheson

Tarsos I y IV sin joroba preapical, notoria, especialmente débil en el tarso IV. Dorso fuertemente estrecho en la parte anterior, con ápex agudo y cónico. Hipostoma con dientes fuertes en la parte media $\mathbf{y}$ pequeños hacia el ápice .... o. brodyi Matheson

8 - Coxa II más grande que la coxa I. Tarsos con un espolón agudo, dorsal y terminal, de modo que la extremidad parece horquillada ...... ................ O. furcosus Neumann Coxa II más pequeña que la coxa I. Tarsos ligeramente angostos hacia la punta. Tres jorobas dorsales en los tarsos de las patas I a III ............ O. turicatus (Dugès) Ornithodoros talaje (Guérin).

Argas talaje Guérin, 1849, Revne Mag. Zool. (2), I, p. 342, Pl., figs. 1.10 ( $\%$ o ; en huésped humano; Casa Vieja de Gastoya en la carretera entre la ciu. dad de Guatemala y Zacapa).
Ornithodoros talaje Dunn, 1923, Amer. Jl. Trop. Med., III, p. 92 (en parte solamente) ; 1927, Jl. of Parasitologie, XIII, p. 177; 1929, Amer. Jl. Trop. Med., IX, p. 507; 1931, Psyche, XXXVIII, p. 170.

Colombia: Barranquilla, en rata gris, Mus nor. vegicus y en casas (Dunn). La he visto procedente de La Dorada, Caldas (Dr. Marco A. Cadena, Col., Junio, 1939). Viotá, Cundinamarca (Brumpt. 1939). Don Matías, Antioquia (Col., Dr. F. Navarro, 1939). Llano Grande, Caldas (E. Osorno, Abril, 1940). Honda, Tolima (Hernando Rey, 1940).

Panamá: Ancón; Ciudad de Panamá; Colón; Chorrera; San Juan (Darling; Jennings y Dunn. Citado por Dunn, 1927).

La distribución y los huéspedes normales de $O$. talaje no se conocen con certeza, debido a la confusión que ha existido entre esta especie y 0 . rudis (=venezuelensis). De acuerdo con Dunn (1929) las larvas se hallan comúnmente en ratas y él asegura que las ninfas y los adultos generalmente atacan roedores y animales grandes y que el hombre es finicamente un huésped de emergencia.

La experimentación ha demostrado que 0 . talaje puede transmitir la Fiebre Recurrente, pero que 0 . rudis es probablemente el principal transmisor de esta enfermedad en el norte de Sur-América, en condiciones naturales.

Los records de Nuttall y Warburton sobre 0 . talaje en Colombia (principalmente en Santana-Tolima), se basaron, por lo menos en parte, en ejemplares de 0 . rudis. Su descripcion se refiere aparentemente a ambas especies (1908, Ticks, I, pp. 59-61).

\section{Ornithodoros rudis Karsch}

Ornithodoros rudis Karsch, 1880, Milth. Munchener Ent. Ver., IV, p. 141 ("New Grenada"; colectado por Goudot).

Ornithoros talaje Dunn, 1923, Amer. Jl. Trop. Med., III, p. 92 (en parte solamente).

Ornithodorus venezuelensis Brumpt, 1922, Précis de Parasitologie, 3rd. Edition, p. 773, fig. 444 (Venezuela y Colombia; en las montañas entre 1000 y 1500 m.). Ruge, 1928, Arch. f. Schiffs-u. Tropenhyg., XXXII, p. 406. Antunes, 1937, Rev. Fac. Medicina, Bogotá, VI, pt. 2, p. 67.

Ornithodoros venezuelensis Dunn, 1927, Jl. of Parasitology, XIII, p. 177; 1929, Amer. Jl. Trop. Med., IX, p. 507.

Colombia.-El Profesor Roberto Franco tiene el honor de haber sido el primer investigador colombiano que encontró que el Ornithodoros rudis Karsch (=venezuelensis Brumpt) es el vector común de la Fiebre Recurrente entre nosotros (*). En esa época lo determinó como Ornithodorus turicata (Franco, Toro y Martínez, 1911, Ses. Científ. del Centenario, Acad. Nac. Med. Bogotá, I, pp. 169-227). M. Roca García (Tesis de grado) siguiendo a Franco, lo determinó también como Ornithodorus turicata (1934).

(*) Al revisar con mayor cuidado la literatura, quien demostro experimentalmente la acertada opinion del Profesor Franco, fue el Dr. Manuel Roca Garefa. (Tesis de Grado, 1934). 


\begin{tabular}{|c|c|c|c|c|}
\hline Departamento & Municipio & $\begin{array}{l}\text { Altura } \\
\text { mts. }\end{array}$ & $\begin{array}{l}\text { Temperatura } \\
\text { media } C^{\circ}\end{array}$ & \\
\hline Antioquia & Antioquia & 709 & 27 & A. Gast G. (1937) \\
\hline$"$ & Barbosa & 1359 & 21 & E. Brumpt (1939) \\
\hline$"$ & Caracoli & 118 & 24 & E. Brumpt (1939) \\
\hline$"$ & Ebéjico & 720 & 22 & L. H. Dunn (1929), A. Gast G. (27-IX-38) \\
\hline$"$ & Medellín & 1538 & 21 & E. Brumpt $(1939)$ \\
\hline$"$ & $\begin{array}{l}\text { Puerto Berrío } \\
\text { (Maceo) }\end{array}$ & 123 & 27 & A. Gast G. (1937) \\
\hline$"$ & San Rafael & 1105 & 23 & E. Osorno (1929) \\
\hline$"$ & Segovia & 900 & 24 & A. Gast G. (1938) \\
\hline$"$ & $\begin{array}{l}\text { Yolombó } \\
\text { (Yali) }\end{array}$ & 1485 & 21 & A. Gast G. (1938) \\
\hline Atlántico & Barranquilla & 4 & 28 & L. H. Dunn (1929) \\
\hline$"$ & Puerto Colombia & 5 & 28 & L. H. Dunn (1929) \\
\hline ” & Soledad & 5 & 28 & L. H. Dunn (1929) \\
\hline Boyacá & Muzo & 927 & 24 & $\begin{array}{l}\text { J. C. Bequaert, E. Osorno (1936), Dr. R. } \\
\text { Franco (1907) }\end{array}$ \\
\hline$"$ & Pauna & 1632 & 20 & E. Brumpt (1939) \\
\hline Caldas & Filadelfia & 1637 & 20 & E. Brumpt, E. Osorno (1939) \\
\hline$"$ & La Virginia & 198 & 33 & E. Brumpt (1939) \\
\hline " & Pereira & 1467 & 21 & E. Brumpt (1939) \\
\hline$"$ & Quimbaya & 1375 & 17 & E. Brumpt (1939) \\
\hline$"$ & Riosucio & 1813 & 19 & E. Brumpt (1939) \\
\hline$"$ & Victoria & 675 & 26 & E. Brumpt, E. Osorno (1939) \\
\hline Cauca & Güapi & 5 & 29 & E. Brumpt, E. Osorno (1939) \\
\hline Cundinamarca & El Peñon & 1310 & 20 & E. Brumpt (1939) \\
\hline$"$ & Fusagasugá & 1746 & 21 & E. Osorno $(1939)$ \\
\hline$"$ & Girardot & 326 & 28 & L. H. Dumn (1929), E. Osorno (1938) \\
\hline$"$ & Guayabal de s. & 1671 & 20 & E. Brumpt (1939) \\
\hline$"$ & Medina & 576 & 27 & L. H. Dunn (1929) \\
\hline$"$ & Paime & 1038 & 25 & E. Brumpt (1939) \\
\hline$"$ & Quipile & 1310 & 20 & E. Brumpt (1939) \\
\hline ” & Sasaima & 1225 & 20 & Dr. M. Roca Gareía (1934) \\
\hline$"$ & Topaipí & 1378 & 20 & E. Brumpt (1939) \\
\hline$"$ & Utica & 503 & 27 & Dr. M. Roca García (1934) \\
\hline$"$ & Vergara & 1570 & 22 & E. Brumpt, E. Osorno (1939) \\
\hline$"$ & Villeta & 842 & 24 & Dr. M. Roca García (1934) \\
\hline$"$ & Viotá & 750 & 25 & E. Brumpt (1939) \\
\hline$"$ & $\begin{array}{l}\text { Yacopí } \\
\text { (Ibama) }\end{array}$ & 1416 & 21 & E. Brumpt (1939) \\
\hline$"$ & Yacopi & 1416 & 21 & E. Brumpt, E. Osorno (1939) \\
\hline Caquetá (Com.) & Florencia & $?$ & 26 & E. Brumpt, E. Osorno (1939) \\
\hline Chocó (Int.) & Istmina & 65 & 25 & $\begin{array}{l}\text { L. H. Dunn (1929), J. C. Bequaert, E. Osor- } \\
\text { no (1937) }\end{array}$ \\
\hline$"$ & Quibdó & 43 & 29 & L. H. Dunn (1929) \\
\hline$"$ & (El Carmen) & & & \\
\hline & Quibdó & 43 & 29 & L. H. Dunn (1929) \\
\hline$"$ & $\begin{array}{l}\text { Quibdó } \\
\text { (Lloró) }\end{array}$ & 43 & 29 & L. H. Dunn (1929) \\
\hline Huila & La Plata & 1054 & 24 & E. Brumpt (1939) \\
\hline Meta & Restrepo & 420 & 26 & J. C. Bequaert, E. Osогno (1936) \\
\hline$”$ & Villavicencio & 498 & 26 & E. Brumpt (1939) \\
\hline Nariño & Barbacoas & 36 & 28 & L. H. Dunn (1929), E. Brumpt (1939) \\
\hline$"$ & Patía & 229 & 20 & E. Brumpt (1939) \\
\hline$"$ & San José & 1912 & 18 & A. Gast $(1940)$ \\
\hline$"$ & Tumaco & 6 & 25 & $\begin{array}{l}\text { L. H. Dunn (1929), E. Brumpt, E. Osorno } \\
\text { (1939) }\end{array}$ \\
\hline Santander & Buearamanga & 1018 & 23 & $\begin{array}{l}\text { L. H. Dunn (1929), E. Brumpt, E. Osorno } \\
(1939)\end{array}$ \\
\hline ” & Girón & 777 & 24 & L. H. Dunn (1929) \\
\hline
\end{tabular}




\begin{tabular}{|c|c|c|c|c|}
\hline Departamento & Municipio & Altura & $\begin{array}{c}\text { Temperatura } \\
\text { media } C^{\circ}\end{array}$ & \\
\hline " & Guadalupe & 1395 & 21 & Dr. M. Roca García (1934) \\
\hline$"$ & Güepsa & 1540 & 22 & Dr. M. Roca García (1934) \\
\hline$"$ & Oiba & 1395 & 20 & E. Brumpt, E. Osorno (1939) \\
\hline$"$ & Rionegro & 590 & 22 & E. Brumpt (1939) \\
\hline$"$ & San Benito & 1428 & 22 & Dr. M. Roca García (1934) \\
\hline$"$ & San Vicente & 692 & 24 & E. Brumpt, E. Osorno (1939) \\
\hline$"$ & Suaita & 1610 & 21 & Dr. M. Roca Gareía (1934) \\
\hline$"$ & $\begin{array}{l}\text { Suaita } \\
\text { (San José) }\end{array}$ & 1610 & 21 & Dr. M. Roca García (1934) \\
\hline Santander (N.) & Cúcuta & 215 & 27 & E. Brumpt, E. Osorno (1939) \\
\hline Tolima & Armero & 421 & 26 & E. Brumpt (1939) \\
\hline$"$ & Chaparral & 880 & 25 & E. Brumpt (1939) \\
\hline ” & Espinal & 438 & 28 & E. Brumpt, E. Osorno (1939) \\
\hline$"$ & Honda & 229 & 31 & E. Brumpt, E. Osorno (1939) \\
\hline$"$ & Ibagué & 1250 & 22 & $\begin{array}{l}\text { L. H. Dunn (1929), E. Osorno (1938), E. } \\
\text { Brumpt (1939) }\end{array}$ \\
\hline$"$ & Icononzo & 1304 & 21 & E. Brumpt (1939) \\
\hline$"$ & Mariquita & 535 & 27 & E. Brumpt (1939) \\
\hline Valle & Buenaventura & 12 & 27 & L. H. Dunn (1929), E. Brumpt (1939) \\
\hline$"$ & Cali & 1003 & 23 & E. Brumpt (1939) \\
\hline$”$ & Palmira & 1085 & 24 & L. H. Dunn (1929), E. Brumpt (1939) \\
\hline$?$ & (Yoto) & $?$ & $?$ & L. H. Dunn (1929) \\
\hline$?$ & (La Vuelta) & $?$ & $?$ & L. H. Dunn (1929) \\
\hline
\end{tabular}

Panamá: La mayor parte de las garrapatas de la Zona del Canal y de Panamá anotadas como 0 . talaje y vectores de Fiebre Recurrente, parece que hayan sido O. rudis (v. L. H. Dunn, 1927).

Brumpt fue el primero que llamó la atención acerca de la confusión predominante, respecto a la identificación de la garrapata que actúa como el vector común de la Fiebre Recurrente en Panamá, Colombia y Venezuela. Separó al verdadero vector, como O. venezuelensis, señalando las principales diferencias con 0 . talaje. La primera descripción detallada de 0 . venezuelensis fue publicada por Ruge en 1928 . Las descripciones previas de 0 . talaje por Neumann (1896, Mém. Soc. Zool. France, IX, p. 34) y por Nuttall y Warburton (1908), se basaron en una mezcla de las dos especies. Además Neumann (1901, Mém. Soc. Zool. France, XIV, p. 269) sinonimiz6 Ornithodoros rudis Karsch con O. talaje y Nuttall y Warburton lo siguieron. Después de leer la descripción de Karsch, conjeturo que él en realidad describió la especie reconocida más tarde por Brumpt. Varios ejemplares de "venezuelensis" procedentes de Colombia, se le remitieron al Profesor P. Schulze, de Rostock, con el objeto de que los comparara con los tipos de Karsch. El Profesor P. Schulze bondadosamente hizo la comparación, llegando a la conclusión de que rudis concordaba con los ejemplares remitidos de Colombia como venezuelensis.

Argas reticulatus Gervais (1849) de Chile, fue anotado por Nuttall y Warburton $(1908$, p. 41) entre las especies "suprimidas". A. C. Oudemans (1936, Kritisch Historisch Overzicht der Acarologic, pt. III, vol. B, p. 794) la consideró idéntica a 0 . talaje, anotando que Neumann vio los ejemplares originales. La descripción de Gervais concuerda mejor con $O$. rudis que con el verdadero $O$. talaje; todavía mejor con las figuras publicadas de O. migonei Brumpt, procedente de Paraguay (1936, Précis de Parasitologie, 5a Edición, II, p. 1209, figs. 665-666).

\section{Familia IXODIDAE \\ IXODES Latreille}

Las especies suramericanas de este género no están todavía suficientemente estudiadas. Se encuentran con más frecuencia en mamíferos de pequeña talla y en aves, que en animales grandes.

Algunas de las especies adquiridas en el curso de las investigaciones sobre Fiebre Amarilla, parece que no están descritas. Es imposible en la actualidad dar una clave práctica para este género.

1 - Ixodes loricatus var. spincsus Nuttall.

El Dr. Bequaert asigna a esta forma, garrapatas colectadas en "chucha real", Metachirus longicaudatus columbianus, procedentes de Restrepo, Int. Meta, por el Dr. J. Boshell M.; otros ejemplares fueron colectados en Didelphys marsupialis, procedentes de Muzo, Depto. Boyacá. He visto ejemplares colectados en Didelphys marsupialis, procedentes de Villavicencio, Int. del Meta.

2 - Ixodes brunneus Koch, variedad.-Neumann. (1904, Arch. de Parasitologie, VIII, p. 454) descrito como una variedad de $I$. brunneus (de Norte América); una hembra colectada en una ave, Ramphocelus coccineus, procedente de Bogotá. Parece que este ejemplar pertenezca a una especie diferente, todavía no descrita. 
3 - Ixodes boliviensis Neumann.-El Dr. Bequaert asigna inciertamente a esta especie de ninfa colectada en Didelphys marsupialis, procedente de Muzo, Depto. Boyacá, por el Dr. J. Boshell M.

4 - Ixodes juvenis Neumann (1899, Mém. Soc.. Zool. France, XII, p. 124) ; ninfa y larva, en Holotropis; ("Nueva Granada"). Los ejemplares fueron colectados en una Iguana, procedente quizá de Colombia. Las especies no se han comprobado, por ser cos adultos desconocidos.

L. H. Duun (1923, Amer. J1. 'Trop. Med., III, p. 94) refiere que una sola hembra de Ixodes ricinus (Linnaeus) fue encontrada en el Río Boquerón, distrito de Panamá.

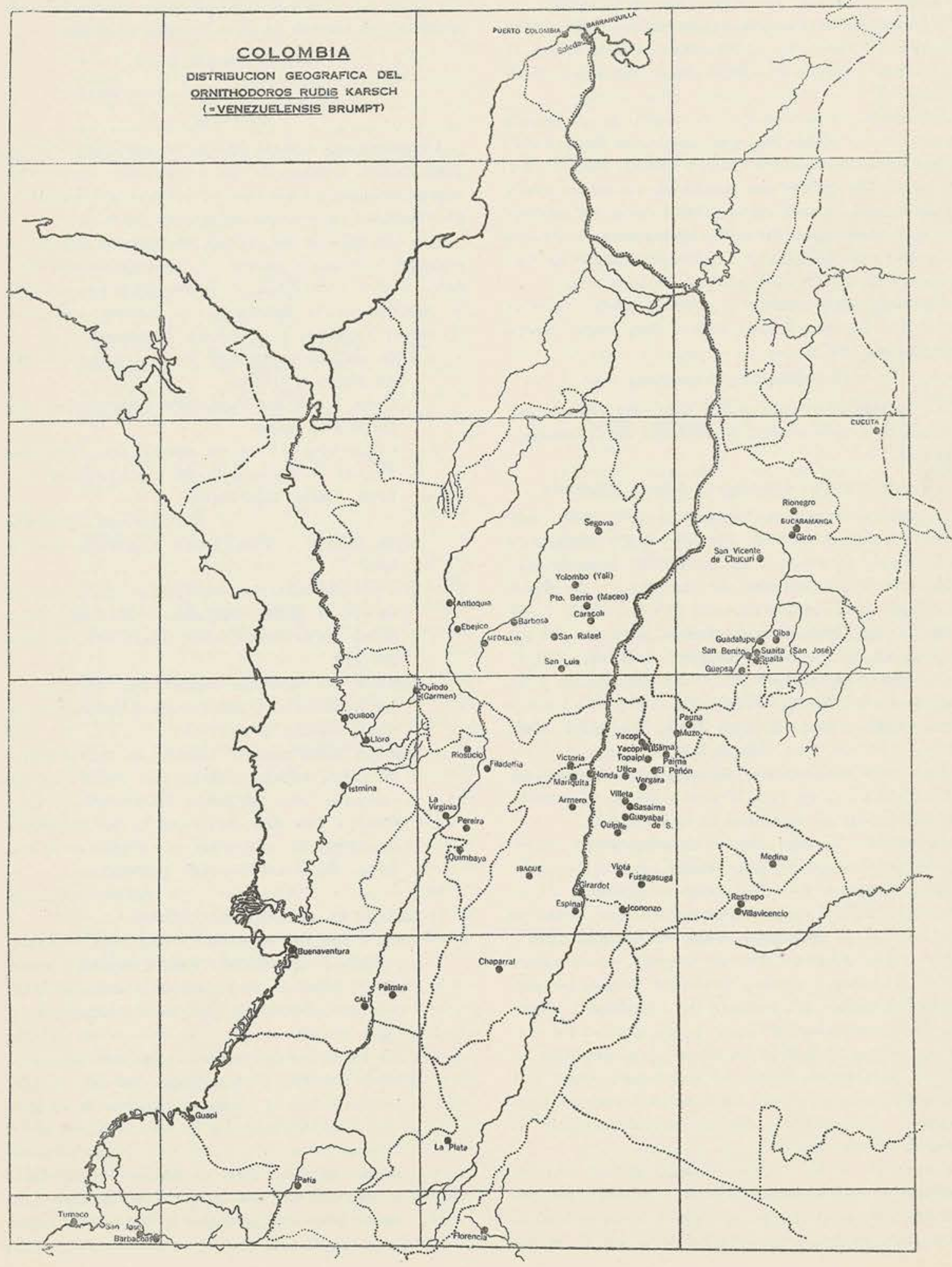




\section{RHIPICEPHALUS Koch}

Parece que haya $\tan$ sólo una especie americana de este género, probablemente introducida por el hombre, siendo así que el huésped común es el perro doméstico.

\section{Rhipicephalus sanguineus (Latreille)}

Ixodes sanguineus Latreille, 1806, Gen. Crust. Ins., I, p. 157 (Francia; sin especificar el huésped).

Rhipicephalus sanguineus Neumann, 1897, Mém. Soc. Zool. France, X, p. 385. Dunn, 1923, Amer. Jl. Trop. Med., III, p. 94; 1929, Amer. Jl. Trop. Med., IX, p. 508.

Colombia.--Una hembra fue colectada en Bogotá en un perro doméstico que, según se dijo, nunca había salido fuera de la ciudad (Dr. J. Boshell M.). Dunn (1929) refiere que es común en varias localidades y que algunas veces infesta casas en Barranquilla y Magangué. He visto ejemplares colectados en perros, procedentes de La Dorada, Depto. de Caldas, por Hernando Rey (1940).

Panamá.-Muy común y prácticamente existe en todos los perros (Dunn, 1923). Río, Bajo Obispo (Neumann, 1897).

\section{DERMACENTOR Koch}

Actualmente se conoce tan sólo una especie perteneciente a este género, procedente de Colombia y Panamá.

\section{Dermacentor (Anocentor) nitens Neumann}

Dermacentor nitens Neumann, 1897, Mém. Soc. Zool. France, X, p. 376, fig. 28 ( $\circ$ ô ; ejemplares procedentes de América sin especificar la localidad; "pueden ser procedentes de Maryland", de Santo Domingo o de Jamaica). (N. Banks, 1908, U. S. Dept. Agric., Bur. Entom. Techn. Ser., No. 15 pp. 50 y 55, Pl. VII, figs. 7 y 10 ( $q$ ô ). Stiles, 1910, U. S. Hygienic Laboratory, Bull. No. 62 , pp. 25 y 63 , figs. $40-43,69,70,81,99,100,118,119$ y 134 (우 하). Dunn, 1923, Amer. Jl. Trop. Med., III, p. 93; 1929, Amer. Jl. Trop. Med., IX, p. 508.

Anocentor colombianus Schulze, 1937, Zoolog. Anzeiger, CXX, p. 24, figs. $1^{\text {a }}$-g y 2 ( ô ô ; Colombia, sin especificar la localidad ni el huésped).

Colombia.-Villeta, Depto. Cundinamarca, en caballos, localizados en las orejas (J. C. Bequaert, 1936) ; Restrepo, Int. del Meta, en caballos (J. C. Bequaert, 1936) ; Villavicencio, Int. del Meta, en caballos (J. C. Bequaert, 1936, E. Osorno, 1939) ; Muzo (Río Minero), Depto. Boyacá, en venado) (Serv. F. Amar.) ; Maní y Trinidad, Depto. Boyacá, huésped humano (J. Boshell M.) ; Medellín, Depto. Antioquia, en caballos (L. H. Dunn. 1929) ; Barranquilla, Depto. Atlántico, en asnos, localizados en las orejas (E. Osorno, 1938). He visto ejemplares procedentes de caballos, de muchas regiones del país. Panamá: es abundante en caballos de cualquier lo. calidad (Dunn, 1923).

Con el Dr. J. C. Bequaert vimos muchos machos, hembras y ninfas procedentes de Colombia, sin que hubiéramos podido encontrar ningún detalle taxonó mico para separarlos de varios lotes de $D$. nitens procedentes de Yucatán, Cuba y Puerto Rico. En cambio, los ejemplares concuerdan en todos los detalles con la descripción y las figuras de Anocentor colombianus. Schulze parece que no haya comparado ejemplares de nitens. El únicamente informa que su especie es "claramente diferente" de nitens, tal como la describe Neumann y la dibujan Banks, Salmon y Stiles, pero sin decir la razón. Que Anocentor deba ser colocado como nombre genérico o subgenérico, es asunto de pura opinión personal.

\section{AMBLYOMMA Koch}

Clave para las Especies Americanas

\section{HEMBRAS}

Propongo las siguientes claves para las especies americanas, muchas de las cuales son imperfectamente conocidas y algunas de las que se encuentran en Colombia parece que no se han descrito.

Las hembras de las siguientes especies son desconocidas o inidentificables: A. quasi-striatum Rondelli, A. Tenellum Koch, A. myrmecophagae Schulze, A. multipunctum Neumann, A. fuscum Neumann, A. torrei Vigueras y A. fulvum Neumann.

1 - Ojos pequeños semiesféricos, contorneados por un surco u órbita.

Coxa I con dos espolones desiguales, el externo más largo.

Coxas II y III con un espolón pequeño. Coxa IV con un espolón largo y puntiagudo (Bolivia, Chile, Argentina) $\ldots \ldots \ldots \ldots \ldots \ldots$ .............. A. parvitarsum Neumann

Ojos planos o ligeramente convejos, sin $6 \mathrm{r}-$ bita ........................ 2

2 - Escudo sin manchas esmaltadas, más o menos oscuro o pálido castaño, o manchado con areas amarillosas. Dentición del hipostoma $3 / 3 \ldots \ldots \ldots \ldots \ldots \ldots \ldots \ldots$

Escudo con manchas esmaltadas (algunas veces indefinidas), con manchas pálidas en un fondo oscuro o viceversa ............6 6

3 - Escudo notoriamente bicolor, la mayor parte de color pálido castaño con lados oscuros castaños, con punteado diseminado y fino. Coxa I con dos espolones cortos, romos y ampliamente separados, el externo es el más largo. Base del capítulo pentagonal ...... ................. A. curruca Schulze

Escudo no notoriamente bicolor ........4 4

4 - Coxa I con dos espolones desiguales, el externo más largo. Escudo sub-triangular, pequeño $(1.5 \mathrm{~mm}$.$) , uniformemente castaño oscu-$ ro, con abundante punteado diseminado y fino. (Brasil) …..... A. parvum Aragão Coxa I con dos espolones cortos, casi iguales, 5

5 - Primer artículo de los palpos con una protuberancia fuerte y roma localizada en el lado ventral y dirigida hacia atrás. Coxas III y IV con un espolón corto. Escudo castaño moreno, con una mancha pálida longitudinal e irregular en cada lado, con abundante punteado pequeño, igualmente distribuído; más toseo hacia las partes laterales (formando 
una depresión corta cerca de cada ojo). (Brasil, Argentina) ..... A. auricularium Conil Primer artículo de los palpos sin protuberancia ventral. Coxas III y IV con dos espolones cortos. Escudo con abundante punteado de tamaño mediano distribuído hacia los lados y escaso en los otros sitios. (Sur América)..$\ldots \ldots \ldots \ldots \ldots$ A. goeldii Neumann

6 - Dentición del hipostoma $4 / 4 \ldots \ldots \ldots .7$ Dentición del hipostoma $3 / 3 \ldots \ldots \ldots \ldots 17$

7 - Coxa I con dos espolones largos y fuertes. Coxas II, III y IV con un solo espolón corto. Cara ventral de los festones con una placa de color oscuro (pelta) y con un tubéreulo pequeño y redondo, en el ángulo posterointerno de cada uno. (Eeuador, Bolivia, Brasil) ............... incisum Neumann

Coxa I con espolones medianos o cortos ...8

8 - Coxas II y IV con dos espolones, el interno algunas veces muy pequeño 0 reducido a una arista fina $\mathrm{y}$ saliente $\ldots \ldots \ldots \ldots$.

Coxas II y IV con un espolón ......... 15

9 - Coxa I con los espolones notoriamente más largos que los de las coxas II, III y IV. Espolones internos de las coxas II, III y IV, muy pequeños, reducidos a finas aristas salien-

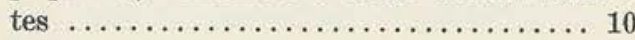

Coxa I con espolones de longitud casi igual a los de las otras coxas ............. 11

10 - Cara ventral de los festones con tubérculos quitinosos en los ángulos posterointernos. Escudo en su mayor parte pálido en fondo oscuro. (Brasil) ..... A. brasiliense Aragão

Cara ventral de los festones sin tubérculos quitinosos en los ángulos posterointernos. Escudo en su mayor parte casi castaño, con una mancha pálida en el ángulo posterior. (Brasil, Bolivia) .....A. scalpturatum Neumann

11 - Escudo cordiforme, con ángulos laterales y posterior ampliamente redondeados .... 12

Escudo triangular o subtriangular, aguzado hacia el ángulo posterior ............ 13

12 - Escudo en su mayor parte de color blanco amarillento con manchas castañas. Espolones internos de las coxas II, III y IV, iguales o ligeramente más pequeños que los externos. Punteado abundante, la mayor parte fino; unos pocos puntos grandes localizados en los ángulos laterales. (Florida) ....... ............... A. tuberculatum Marx

Escudo en su mayor parte de color oscuro castaño, con una mancha pálida e irregular a cada lado, cerca del ojo. Espolones internos de las coxas II, III y IV anchos, en forma de arista. Punteado de tamaño mediano, casi igual y uniformemente distribuído. (Guayana)..$\ldots \ldots \ldots \ldots$. brimonti Neumann

13 - Espolones de la coxa I notoriamente separados, los de las coxas II, III y IV más o menos juntos o fusionados en la base. (Panamá)..$\ldots \ldots \ldots \ldots$ A. crassum Robinson
Espolones de todas las coxas notoriamente separados en la base ................ 14

14 - Los espolones internos de todas las coxas apro. ximadamente del mismo tamaño y casi tan anchos como los espolones externos. Escudo triangular-cordiforme, aproximadamente $\tan$ largo como ancho. (Sur América) ....... .................. humerale Koch

Los espolones internos de las coxas disminn. yen gradualmente de tamaño de la I a la IV; los de las coxas III y IV son más pequeños que los externos. Escudo triangular, ligeramente más ancho que largo (Guatemala) $\ldots \ldots \ldots \ldots \ldots \ldots \ldots \ldots \ldots$ A. sabanerae stoll

15 - Espolones de las coxas II y III tan largos como anchos o por lo menos no mucho más anchos que largos, sin forma de arista. Escudo vagamente ormamentado, amarillo pálido en la mayor parte, con abundante punteado. (Brasil) ......... pictum Neumann

Espolones de las coxas II y III en forma de aristas anchas y salientes, mucho más anchas que largas ................ 16

16 - Escudo subcordiforme, con los ángulos laterales y posterior igualmente redondeados, grande $(3.5 \times 4.2 \mathrm{~mm}$.), la mayor parte de color oscuro castaño, con manchas pálidas. (Guayana) ........... guianense Neumann

Escudo triangular, con los ángulos laterales bien diseñados, pequeño $(2.1 \times 2.4 \mathrm{~mm}$. $)$, la mayor parte de color amarillo pálido, con manchas oscuras, rojizo castañas. (Paraguay, Brasil) ........... cooperi Nuttall y Warburton

17 - Coxa $I$ con el espolón interno muy pequeño o vago, el externo bien desarrollado. Sin tubérculos quitinosos en los ángulos posterointernos de la cara ventral de los festones 18

Coxa I con el espolón interno bien desarrollado, aunque algunas veces es mucho más corto que el externo ............... 21

18 - Coxa IV con un espolón largo y agudo. Coxas II y III con espolones cortos y triangulares. Escudo amarillo pálido, manchado con oscuro castaño especialmente a lo largo de los márgenes y de dos fajas medianas. (Argentina) .......... Altiplanum Dios (probablemente $=$ furcula Dönitz) .

Coxa IV con un espolón muy corto y triangular ......................... 19

19 - Coxas II y II con dos espolones pequeños y triangulares, el interno siempre más pequeño $\mathrm{y}$ algunas veces rudimentario en la coxa III. Escudo la mayor parte castaño pálido, con manchas esmaltadas reducidas, en las áreas escapulares y en el ángulo posterior. con escaso punteado grande en la parte anterolateral, y pequeño en los otros sitios.

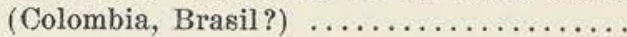
............ A. deminutivum Neumann

Cosas II y III con un espolón muy pequeño, más o menos de forma triangular, a menudo 
casi rudimentario. Punteado uniformemente diseminado sobre el escudo .........20

20 - Escudo blanco, con una mancha castaña estrecha $y$ mediana en el quinto posterior, con punteado abundante de tamaño mediano (Cuba) .......... A. albopictum Neumann Escudo con muchas manchas oscuras en fondo pálido, la mancha castaña mediana empieza en el borde anterior, con abundante punteado fino (Norte América y Sur América).$\ldots \ldots \ldots \ldots \ldots \ldots$ A. maculatum Koch

21 - Coxa I con dos espolones muy desiguales, el interno cuando más dos tercios de la longitud del externo ................. 22

Coxa I con los espolones de longitud igual o casi igual ................... 32

22 - Coxas II y III con un espolón en forma de una arista ancha y saliente, mucho más ancha que larga .................. 23

Coxas II y III o con dos espolones (el interno a menudo muy pequeño) o con un espolón tan largo como ancho y no mucho más ancho que largo (sin forma de arista) ...28

23 - Escudo castaño oscuro, con una mancha esmaltada y nítida en el ángulo posterior. Cara ventral de los festones sin tubéreulos quitinosos en los ángulos postero-internos. (Norte América) ...A. americanum (Linnaeus)

Escudo como norma, en su mayor parte pálido con manchas oscuras. Cara ventral de los festones con un pequeño tubérculo quitinoso en el ángulo postero-interno de cada uno, 24

24 - Areas porosas de la base del capítulo de forma circular ................... 25

Areas porosas de la base del capítulo de forma oval, divergentes en la parte anterior .. 26

25 - Escudo tan ancho como largo, liso y brillante, con escaso punteado pequeño en el área mediana y grande en las áreas escapulares. Peritrema más ancho que largo, con una prolongación dorsal, notoria. (México, Trinidad) ................ mixtum Koch

Escudo ligeramente más ancho que largo, con abundante punteado de tamaño mediano igualmente distribuído, también en el área mediana. Peritrema aproximadamente tan ancho como largo. (Guayana, Brasil) .... .............. cajennense Fabricius

26 - Escudo pequeño, triangular, con las manchas esmaltadas a menudo muy reducidas. Coxa I con los espolones casi iguales en longitud. Palpos más largos que el hipostoma. (Brasil) ............. sculptum Berlese

Escudo grande, triangular. Coxa I con espolones muy desiguales. Palpos ligeramente más cortos que el hipostoma ........... 27

27 - El área esmaltada blanca cubre la mayor parte del escudo (excepto a lo largo de los márgenes postero-laterales). (Paraguay) ..... .................. A. tapiri Rondelli

El área esmaltada blanca mucho más reducida, la mayor parte del escudo manchada con castaño. (Paraguay) A. finitimum Rondelli (A. pecarium Dunn y A. tapirellum Dunn coinciden con 24 y parece que sean cercanas a finitimum).

28 - Coxa I con un espolón interno plano y ancho. Base del capítulo muy corta en relación a su anchura, algo proyectada hacia los lados. Palpos largos y delgados. Ojos planos lateralmente. (Brasil, Guayana) ............ ............. mantiquirense Aragão

Coxa I con un espolón interno estrecho ...29 29

29 - Coxas II y III con dos espolones triangulares, el interno de la coxa III a menudo mncho más pequeño y algunas veces rudimentario. Palpos largos y delgados. Ojos lateralmente no abombados .............. 30

Coxas II y III con un espolón más o menos triangular. Palpos cortos y gruesos. Ojos algo abombados fuera del margen del escudo $\ldots \ldots \ldots \ldots \ldots \ldots \ldots \ldots \ldots . . . \ldots \ldots$

30 -- Coxa I con los dos espolones casi iguales en longitud. Coxas II, III y IV con dos espolones, el interno de la coxa IV algunas veces muy pequeño. Manchas esmaltadas del escudo reducidas generalmente a una mancha cerca del ángulo posterior y a una faja lateral cerca de cada ojo, con escaso punteado muy grande en la parte anterior de los lados y fino en los otros sitios. (Norte América y Sur América) $\ldots \ldots \ldots \ldots \ldots \ldots$. dissimile Koch

Coxa I con dos espolones muy desiguales. Coxas III y IV generalmente con un espolón o con el espolón interno de la coxa III muy pequeño. Manchas esmaltadas del escudo más extendidas, con escaso punteado muy grande en la parte anterior de los lados y fino en los otros sitios. (Colombia, Brasil?) ........ A. deminutivum Neumann

31 - Coxa IV con un espolón largo, delgado y agudo. La mayor parte del escudo pálida, con margen oscuro castaño, con unas pocas manchas y dos bandas longitudinales de color castaño que llegan al margen posterior. (Argentina) ........... A. altiplanum Dios

Coxa IV con un espolón más corto y más triangular. La mayor parte del escudo pálida, casi castaño a lo largo del margen, con una banda o mancha mediana de color oscuro en la mitad posterior y dos bandas cortas y oscuras en la mitad anterior (que no llegan al margen posterior). (Argentina) ........ ................... furcula Dönitz

(Estas dos especies son de dudosa distinción).

32 - Coxa I con espolones largos .......... 33

Coxa I con espolones de tamaño mediano o cortos ....................... 40

33 - Coxa I con espolones delgados ......... 34

Coxa I con espolones fuertes ........... 36

34 - Coxa I con espolones moderadamente largos, que dividen la coxa hasta cerca de la mitad. La mayor parte del escudo castaña, con man- 
chas esmaltadas grandes, con punteado pequeño que es muy escaso en el área mediana.

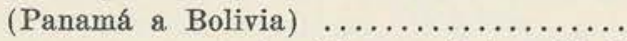
.............. oblongoguttatum Koch

Coxa I con espolones muy largos y agudos, que dividen la coxa más allá de la mitad .... 35

35 - A. ovale Koch, A. fossum Neumann y A. striatum Koch, que todavía no se pueden separar por la hembra.

36 - Coxa I con dos espolones medianamente largos y fuertes, que no dividen la coxa hasta cerca de la mitad. Escudo pequeño $(2.2 \times$ $2.5 \mathrm{~mm}$.), liso y brillante, de color castaño claro, con unas pocas manchas esmaltadas borrosas y con abundante punteado pequeño, diseminado. (Brasil, Paraguay) ...... ..................... pacae Aragão

Coxa I con dos espolones largos y aplanados, que dividen la coxa hasta más allá de la mitad. Escudo claramente ornamentado, con abundante punteado tosco .......... 37

37 - La mayor parte del escudo pálida, con manchas castañas estrechas. Base del capítulo rectangular. (México a Bolivia) .......... ........................elebs Neumann

La mayor parte del escudo clara o castaña oscura con unas pocas manchas pálidas esmaltadas ......................... 38

38 - Escudo con una mancha pálida en el ángulo posterior y otra irregular, pálida, en forma de $Y$, en cada lado. Base del capítulo casi rectangular. El espolón de la coxa IV ligeramente más largo que ancho. Segundo artículo de los palpos con una arista oblicua en la cara dorsal. (Guatemala a Colombia) .... ................ nodosum Neumann

Coxa IV con el espolón mucho más largo que ancho. Base del capítulo pentagonal o subtriangular .................... 39

39 - Escudo con dos hoyuelos cortos, profundos y ovoides en la parte anterior, de cada uno de los cuales parte un surco superficial que se extiende hacia la parte posterior. Segundo artículo de los palpos con una arista ligeramente oblicua en la cara dorsal. (Sur América) ......... A. calcaratum Neumann

Escudo con dos surcos divergentes, largos y superficiales, continuados hacia atrás. Segundo artículo de los palpos sin arista oblicua en la cara dorsal. (Brasil) $\ldots \ldots \ldots \ldots$ ............... A. leucozomum Schulze

40 - Coxa IV con dos espolones, el interno a menudo muy pequeño o vago $\ldots \ldots \ldots \ldots .41$

Coxa IV con un espolón ............ 46

41 - Cuerpo cubierto con pelos largos, suaves y blanquecinos detrás del escudo ....... 42

Cuerpo glabro o casi glabro o con pelos blanquecinos cortos, fuertes y comprimidos contra el cuerpo .................. 43

42 - Los pelos forman pequeñas brochas en el margen, especialmente hacia atrás. Escudo ampliamente cordiforme, más ancho que largo.
Palpos notoriamente dilatados más allá de la base. (Galápagos).. A. hirtum Neumann

Los pelos no forman brochas. Escudo triangular, aproximadamente tan largo como ancho. Palpos delgados en toda la extensión. (Galápagos) ............. A. pilosum Neumann

43 - Escudo con la mayor parte pálida, amarillo dorado, con unas pocas manchas castañas; cordiforme, con abundante punteado fino $y$ escaso punteado grueso diseminado. (Argentina).$\ldots \ldots \ldots \ldots \ldots$ A. testudinis Conil

Escudo con la mayor parte de color oscuro, con menos manchas pálidas ......... 44

44 - Cuerpo con numerosos pelos blanquecinos, cortos, fuertes y comprimidos contra el cuerpo, detrás del escudo. Escudo cordiforme, con abundante punteado profundo y de tamaño mediano, igualmente distribuído. (Galápagos) ............... williamsi Banks

Cuerpo glabro o casi glabro detrás del escudo $\ldots \ldots \ldots \ldots \ldots \ldots \ldots \ldots \ldots \ldots \ldots .45$

45 - Escudo subtriangular, con escaso punteado muy grande (la mayor parte en los lados de la mitad anterior). Espolones de las coxas, más estrechos y más puntiagudos. (Norte América y Sur América). A. dissimile Koch

Escudo cordiforme, con abundante punteado tosco, distribuído con más uniformidad. Espolones de las coxas más anchos y más parecidos a aristas; el espolón interno de la coxa I más ancho que el externo. (Brasil)... ................. rotundatum Koch

46 - Base del capítulo casi triangular ...... 47

Base del capítulo rectangular ........ 49

47 - Escudo oval alargado, casi en forma de losange, más largo que ancho, oscuro castaño con manchas esmaltadas más bien borrosas, con abundante punteado más bien pequeño e igualmente distribuído. Hipostoma muy largo, lanceolado, que se adelgaza hacia la punta. (Sur América) ...... A. longirostre Koch

Escudo triangular o cordiforme, más ancho que largo o tan largo como ancho. Escudo con manchas esmaltadas más nítidas y con punteado tosco, más bien irregular .... 48

48 - Escudo más o menos cordiforme, con un par de surcos ovales y profundos en la parte anterior (cerca de la base del capítulo). Hipostoma espatulado. Tarsos bruscamente atenuados hacia las extremidades (Sur América) $\ldots \ldots \ldots \ldots \ldots \ldots$ A. varium Koch

Escudo más o menos cordiforme, con dos sur$\cos$ anteriores profundos y estrechos, que se continúan hacia la parte posterior a la manera de depresiones superficiales. Hipostoma subagudo hacia la extremidad. Tarsos gradualmente atenuados hacia las extremidades (Sur América) .. A. geayi Neumann

49 - Primer artículo de los palpos con una protuberancia retrograda, fuerte y roma en la cara ventral $\ldots \ldots \ldots \ldots \ldots \ldots \ldots \ldots, 50$ 
Primer artículo de los palpos sin protuberancia retrógrada en la cara ventral ..... 51

50 - Escudo rojizo castaño, vagamente ornamentado con manchas pálidas, pero sin manchas esmaltadas, con dos surcos estrechos y curvos en la parte anterior, que llegan cerca de la base del capitulo. (Brasil, Argentina). ................. Auricularium Conil

Escudo amarillose castaño con manchas esmaltadas nítidas, con dos hoyuelos profundos y piriformes en la parte anterior que no llegan a la base del capítulo. (Brasil, $\mathrm{Pa}$ raguay) ......... pseudoconcolor Aragão

51 - Coxas II y III con dos espolones, el interno a menudo muy pequeño .............. 52

Coxas II y III con un espolón ......... 53

$52-A$. dissimile Koch, A. cruciferum Neumann. (Véase número 30 ).

53 - Coxa I con dos espolones casi iguales, dos veces tan largos como anchos. Coxas II y III con un espolón aplanado, en forma de arista, más ancho que largo. Surcos anteriores medianos del escudo, cortos, profundos y ondulados. (Venezuela) A. bispinosum Neumann

Coxa I con dos espolones pequeños, que no son más largos que anchos. Coxas II y III con un espolón más pequeño, sin forma de aris-

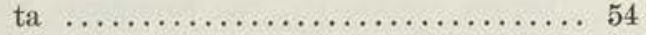

54 - Escudo con numeroso punteado superficial, más bien de tamaño desigual, castaño oseuro, con manchas esmaltadas irregulares y borrosas; un par de surcos anteromedianos y ovalados, en forma de hoyuelos detrás de los cuales hay un par de surcos profundos que se extienden hasta cerca del margen posterior. (Galápagos) .. A. darwini Hirst

Escudo con punteado de tamaño casi igual, de profundidad moderada, regulamente distribuído; un par de surcos profundos y curvos en la parte anterior, que se continuan en la parte posterior en forma de depresiones superficiales .................. 55

55 - Escudo castaño oscuro, vagamente ormamentado con manchas esmaltadas pálidas. Ojos vagos. (Galápagos) .. A. boulengeri Hirst

Escudo con marcas nítidas castañas oscuras en un fondo esmaltado pálido. Ojos nítidos, de tamaño mediano. (Centro y Sur América)..$\ldots \ldots \ldots \ldots$ A. scutatum Neumann

\section{MACHOS}

Los machos de las siguientes especies son desconocidos 0 inidentificables: $A$. bispinosum Neumann, A. scalpturatum Neumann, A. pilosum Neumann, $A$. rotundatum Koch (garrapata partenogenética) y A. cordiferum Neumann.

1 - Ojos pequeños, semi-esféricos, contorneados por un surco deprimido u órbita. Coxa IV con una espina larga. Dentición del hipostoma $3 / 3 \ldots \ldots$..... parvitarsum Neumann

Ojos planos o tan ślo ligeramente convejos, sin órbita
2 - Surco marginal completo, continno a lo largo de todos los festones. Dentición del hipostoma $3 / 3 \ldots \ldots \ldots \ldots \ldots \ldots \ldots \ldots$

Surco marginal o ausente o incompleto (nunca continuo a lo largo de todos los festones en el margen posterior ............ 23

3 - Coxa I con dos espolones largos y continuos, iguales o casi iguales en longitud ..... 4

Coxa I o con espolones cortos o desiguales en longitnd $\ldots \ldots \ldots \ldots \ldots \ldots \ldots \ldots . \ldots \ldots$

4 - Escudo alargado oval, más de vez y media más largo que ancho. Coxa I con espolones muy largos y agudos, mucho más largos que el espolón de la coxa IV ............. 5

Escudo ancho oval, menos de vez y media más largo que ancho, escasamente ornamentado con fajas o manchas pálidas, la mitad anterior no simula el eseudo de la hembra. Coxa I con espolones más cortos aproximadamente tan largos como el espolón de la coxa IV. 7

5 - Espolón externo de la coxa I recto. Escudo con manchas pálidas esmaltadas, muy reducidas, la mayor parte localizadas hacia los lados, sin simular el escudo de la hembra. (Paraguay) ... A. quasi-striatum Rondelli

Espolón externo de la coxa I con la punta ligeramente encorvada hacia afuera ....6 6

6 - Escudo con manchas pálidas esmaltadas bien desarrolladas, que simulan en la parte anterior, el escudo de la hembra. Punteado-tosco, la mayor parte localizado en la parte posterior del escudo y en el surco marginal ................... ovale Koch

Escudo con pocas manchas pálidas esmaltadas, que no simulan en la parte anterior el escudo de la hembra. Punteado tosco, más igualmente distribuído. A. fossum Neumann

7 - Coxa I con espolones anchos y aplanados. Escudo cubierto uniformemente con punteado abundante y grande, aún sobre los festones ................. coelebs Neumann

Coxa I con espolones delgados y agudos. Escudo con escaso punteado fino, localizado en ciertas áreas; en los festones no hay punteado .......... A. oblongoguttatum Koch

8 - Escudo con los lados notoriamente deprimidos entre el ojo y el segundo festón y con una arista en forma de quilla en el área posteromediana; la mayor parte de color más oscuro y castaño pálido, el esmaltado pálido localizado en las áreas escapulares, con unas pocas manchas medianas; escaso punteado superficial distribuído únicamente en las áreas esmaltadas. Coxa I con dos espolones de longitud media, el externo ligeramente más largo y más delgado; coxas II y III con un espolón ancho, en forma de arista; coxa IV con un espolón puntiagudo, moderadamente largo .. A. pecarium Dunn

Escudo sin los lados deprimidos y sin arista en forma de quilla en el área posteromediana $\ldots \ldots \ldots \ldots \ldots \ldots \ldots \ldots \ldots \ldots . \ldots \ldots$ 
9 - Coxa I con el espolón externo largo, delgado y puntiagudo $\ldots \ldots \ldots \ldots \ldots \ldots \ldots \ldots, 10$

Coxa I con el espolón externo corto o mediano y romo. Coxa IV con un espolon corto y romo ....................... 19

10 - Coxa IV con un espolón corto. Coxa I con el espolon interno muy corto. Escudo desornamentado ............... parvum Argão

Coxa IV con un espolón largo y delgado. Escudo con manchas esmaltadas ........ 11

11 - Coxa I con el espolón interno muy pequeño, a menudo escasamente visible. Protarsos II a IV con un par de espinas prominentes en la extremidad distal $y$ hacia la superficie ventral. Escudo alargado, oval, con fajas esmaltadas más o menos longitudinales; punteado abundante pero más bien pequeño y la mayor parte localizado en las áreas esmaltadas ............ A. maculatum Koch

Coxa I con el espolón interno bien desarrollado, sobrepasa la mitad del espolón externo ......................... 12

12 - Escudo con la mayor parte de color oseuro, con unas pocas manchas pequeñas esmaltadas, la mayor parte hacia el margen. Los festones no son salientes hacia atrás. Vientre sin placas .. A. americanum (Linnaeus)

Escudo con áreas levantadas de color oscuro, las manchas esmaltadas más extendidas, a menudo simulan en la parte anterior el escudo de la hembra ................. 13

13 - Vientre con placas pequeñas endurecidas frente a los festones, los cuales llevan peltae levantados $\ldots \ldots \ldots \ldots \ldots \ldots \ldots \ldots \ldots . \ldots \ldots$

Vientre sin placas ni peltae .......... 18

14 - Festones largos y estrechos, todos por lo menos dos veces más largos que anchos ... 15

Festones de anchura normal casi todos menos de dos veces más largos que anchos ... 17

15 - Escudo más alargado, oval (más largo que ancho), en su mayor parte con manchas esmaltadas ........ cajennense (Fabricius)

Escudo más ovalado (aproximadamente tan largo como ancho), con menos manchas esmaltadas (predomina el color oscuro more-

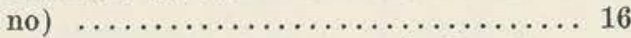

16 - A. finitimum Rondelli, A. tapirellum Dunn.

17 - Escudo lustroso, la mayor parte liso, con escaso punteado en las áreas pálidas. Peritrema en forma de coma, con un prolongamiento dorsal largo ......... A. mixtum Koch

Escudo casi uniformemente cubierto con abundante punteado grande. Peritrema alargado, oval, con un prolongamiento dorsal corto .............. A. tapiri Rondelli

18 - Festones largos y estrechos, todos por lo menos dos veces más largos que anchos. Las áreas esmaltadas del escudo más reducidas, no se extienden en la parte anterior hacia las áreas escapulares .. A. sculptum Berlese

Festones de anchura normal, casi todos menos de dos veces más largos que anchos. Las áreas esmaltadas del escudo más extendidas ; se extienden en la parte anterior hacia las áreas escapulares. (México) ............... ................... A. tenellum Koch

19 - Primer segmento de los palpos sin espolón retrógrado en el lado ventral. Base del capítulo trapezoidal. Coxa I con el espolón interno corto. Vientre con placas endurecidas delante de los festones. Escudo ancho, oval, mucho más ancho hacia atrás, con manchas esmaltadas extensas, uniformemente cubierto con abundante punteado desigual ...... ..................... geayi Neumann

Primer segmento de los palpos con un espolón retrógrado, ancho, plano y romo en el lado ventral. Base del capítulo rectangular. Coxa I con dos espolones cortos casi iguales. Vientre $\sin$ placas endurecidas ..........20

20 - Escudo uniformemente amarilloso moreno, sin manchas esmaltadas, casi liso, con escaso punteado superficial cerca del margen anterior. Festones largos y estrechos, por lo menos dos veces más largos que anchos, los externos encorvados ..... A. curruca Schulze

Escudo o con áreas esmaltadas, o claramente punteado. Festones de forma normal ... 21

21 - La mayor parte del escudo esmaltada, con manchas y rayas nítidas, de color moreno, casi uniformemente cubierto con abundante punteado grande. Coxas II y III con un espolón en forma de arista ................ ......... cooperi Nuttall y Warburton

La mayor parte del escudo, rojiza o amarillosa morena, con unas pocas áreas pequeñas esmaltadas, algunas veces vagas. Coxas II y III con un espolón estrecho, triangular o en forma de espina $\ldots \ldots \ldots \ldots \ldots \ldots .22$

22 - Escudo oscuro, rojizo moreno, con manchas esmaltadas muy pequeñas y a menudo vagas; con abundante punteado pequeño, igualmente distribuido, más tosco hacia los lados en la parte anterior ... A. auricularium Conil

Escudo amarillento moreno, con una serie de manchas nitidas esmaltadas, hacia la periferia; punteado muy fino, escaso y más tosco, formando una línea irregular que continúa el surco marginal hacia la parte anterior ......... A. pseudoconcolor Aragão

23 - Surco marginal casi completo o nútido únicamente hacia los lados. Dentición del hipostoma $3 / 3 \ldots \ldots \ldots \ldots \ldots \ldots \ldots \ldots \ldots \ldots \ldots$

Surco marginal rudimentario en toda la extensión $o$ ausente . ................ 29

24 - Vientre con placas endurecidas prominentes, delante de los festones. Coxa I con dos espolones cortos y puntiagudos, el interno $\mathrm{mu}$ cho más pequeño; coxa IV con un espolón muy corto. Escudo más bien escasamente ornamentado con manchas o fajas esmaltadas ; con abundante punteado pequeño, pero profundo; el surco marginal se extiende a lo 
largo de los dos primeros festones ........ ................ longirostre Koch Vientre sin placas endurecidas ........25

25 - Surco marginal interrumpido en cada lado, finicamente frente al quinto festón. Coxa IV con un espolón largo y puntiagudo . . . . 26

Surco marginal sin extenderse hacia la parte posterior, más allá del primer festón ... 27

$26-A$. Furcula Dönitz.

A. altiplanum Dios (¿surco marginal completo).

27 - Coxa IV con un espolón largo, delgado y agudo, tan largo como la coxa; coxa I con dos espolones ligeramente desiguales, el interno algo aplanado y más corto. Palpos con un apéndice romo en la cara dorsal de la base del tercer artículo; base del segundo artículo cortada oblicuamente en la cara dorsal ... ................ brasiliense Aragão

Coxa IV con un espolón triangular, mucho más corto que la coxa ............. 28

28 - Coxa I con dos espolones iguales y paralelos, el interno más ancho y aplanado. Palpos con un apéndice romo en la cara dorsal de la base del tercer artículo; la base del segundo artículo no está cortada oblicuamente en la cara dorsal. Escudo corto, oval, no es deprimido delante de los festones, que son muy cortos; la mayor parte de color oseuro o moreno pálido, con manchas esmaltadas disociadas. (Surinam)

........... A. myrmecophagae Schulze

Coxa I con dos espolones largos y paralelos, el interno más romo, pero no mucho más ancho que el externo. Escudo alargado oval, deprimido delante de los festones, que son por lo menos tan largos como anchos; la mayor parte esmaltada, con manchas oscuras morenas ............. A. striatum Koch

29 - Dentición del hipostoma $4 / 4 \ldots \ldots \ldots \ldots 30$

Dentición del hipostoma $3 / 3 \ldots \ldots \ldots . .38$

30 - Coxa IV con dos espolones, el interno a menudo pequeño $\ldots \ldots \ldots \ldots \ldots \ldots \ldots \ldots . \ldots \ldots$

Coxa IV con un espolón ............. 34

31 - La mayor parte del escudo mate, blanco amarillento, con una serie de manchas oscuro castañas bien definidas. Los surcos que dividen los festones son muy cortos. Punteado

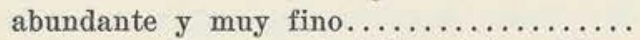
................ A. tuberculatum Marx

La mayor parte del escudo castaña, con manchas pequeñas esmaltadas, dispersas, algunas veces circunscritas a las áreas escapulares. Los surcos que dividen los festones son de longitud normal ............... 32

32 - Manchas esmaltadas reducidas a dos manchas estrechas, en las áreas antero-laterales, que se extienden ligeramente más allá de los ojos. Coxa I con dos espolones anchos, planos y redondeados, como los de la coxa II ...... ................. crassum Robinson
Manchas esmaltadas dispersas, no están circunscritas a las áreas antero-laterales ... 33

33 - Coxa I con dos espolones anchos, planos y redondeados, como los de la coxa II ........ ................ A humerale Koch

Coxa I con dos espolones alargados, agudos, de tamaño mediano y mucho más fuertes que los de la coxa II ........A. sabanerae Stoll

34 - Coxa I con dos espolones contiguos, largos, fuertes y aplanados, iguales o casi iguales, que dividen la coxa hasta cerca de la base; coxa IV con un espolón largo y delgado; coxas II y III con un espolón ancho y en forma de arista, ligeramente dividido por una ranura curva. Escudo de color oscuro, desornamentado, con abundante punteado tosco-densamente aglomerado en ciertas áreas ........ A. multipunctum Neumann

Coxa I con dos espolones cortos o medianos, que dividen la coxa cuando más hasta la

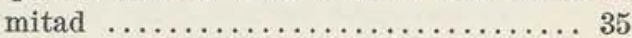

35 - Coxas II y III con un espolón ancho, en forma de arista, mucho más ancho que largo; coxa IV con un espolón muy corto, más corto que la coxa. Escudo oscuro rojizo castaño, desornamentado, con abundante punteado grande, igualmente distribuído ........... .................. goeldii Neumann

Coxas II y III con un espolón corto, tan ancho o ligeramente más ancho que largo, sin forma de arista ................. 36

36 - Coxa I con un surco profundo, transversal, que separa la porción anterior; espolones cortos y aplanados. Coxa IV con un espolon corto y triangular. Escudo sin manchas esmaltadas, la mayor parte de color pálido u oscuro castaño, con escaso punteado disperso, grande y profundo. Vientre con pequeñas placas endurecidas, delante de los festones .......... A. fuscum Neumann

Coxa I sin surco transversal que la divida. 37

37 - Escudo con manchas esmaltadas nítidas, con abundante punteado profundo y casi igual, parcial e igualmente distribuído. Coxa IV con un espolón moderadamente largo y puntiagudo .......... A. guianense Neumann

Escudo vagamente ornamentado con manchas esmaltadas, con abundante punteado pequeño y profundo, confluente en algunos sitios. Coxa IV con un espolon corto y más bien romo ................ pictum Neumann

38 - Coxa IV con dos espolones, el externo más largo, el interno a menudo pequeño . . . 39

Coxa IV con un espolón interno, el externo ausente ..................... 44

39 - Periferia del cuerpo con pelos largos, blanquecinos, a menudo en brochas formadas de dos o tres pelos. Festones claramente definidos, por lo menos tan largos como anchos. La mayor parte del escudo de color blanco amarillento con manchas pálidas castañas, que simulan el escudo de la hembra en la parte 
anterior; punteado abundante profundo, disperso en las áreas pálidas ............. ................ Airtum Neumann

Periferia del cuerpo sin pelos largos, o con pelos gruesos comprimidos contra el cuerpo, o casi glabra ............... 40

40 - Escudo subcircular, casi tan ancho como largo, con hombros obtusos, con depresiones o surcos nítidos que separan un falso escudo de la hembra, con una raya mediana y posterior levantada y áreas laterales e irregulares salientes; la mayor parte cubierta con abundante punteado de tamaño mediano, profundo y discreto. Límites de los festones, escasamente marcados hacia atrás ........ ................ A. williamsi Banks

Escudo ni subcircular, ni con depresiones nítidas, ni con áreas salientes ........ 41

41 - Escudo tan ancho como largo, pálido castaño, con una raya esmaltada grande, continua, en cada lado; con punteado muy fino, uniformemente distribuído. Vientre con placas endurecidas delante de los festones, que están bien delimitados y dorsalmente son tan anchos como largos. (Cuba) .............. ................... torrei Vigueras

Escudo o casi desornamentado o las áreas esmaltadas no forman rayas laterales .... 42

42 - Escudo con la mayor parte de color pálido castaño, con unas pocas manchas esmaltadas a lo largo del margen, en los festones y cerca de ellos, hacia la parte posterior; punteado muy escaso, disperso en la mitad posterior y cerca del margen, casi ausente en el área antero-mediana ................ ............ A. deminutivum Neumann

Escudo con áreas esmaltadas más extensas; punteado desigual, parte muy grande y profundo ........................4 43

43 - Escudo la mayor parte con esmaltado amari110 dorado, con escasas manchas pálidas castañas; punteado de dos tamaños, parcial e igualmente diseminado. Festones cortos, cuando más tan largos como anchos ...... ................. A. testudinis Conil

Escudo con manchas de color rojizo castaño muy extensas, en un fondo esmaltado pálido amarillento; el punteado no está igualmente distribuído, casi falta en el área antero-mediana. (Florida, C. y S. América) ....... ................. dissimile Koch

44 - Coxa I con dos espolones largos, anchos, aplanados, contiguos, iguales o casi iguales .. 45

Coxa I o con espolones cortos o notoriamente desiguales en longitud $\ldots \ldots \ldots \ldots \ldots \ldots 49$

45 - Coxa IV con un espolón largo y delgado, tan largo como la coxa .............. 46

Coxa IV con un espolón corto o mediano, más corto que la coxa ............... 48

46 - Escudo completamente oscuro, sin manchas esmaltadas; punteado abundante, muy tosco, aglomerado en ciertas áreas. Coxas II y
III con un espolón ancho, en forma de arista, más o menos dividido por una ranura curva........... multipunctum Neumann

Escudo claramente ornamentado con manchas esmaltadas pálidas en fondo oscuro; punteado abundante, grande, más bien igualmente distribuído. Coxa II y III con un espolón corto, moderadamente ancho $\mathrm{y}$ sin forma de arista ................... 47

47 - Base del capítulo irregularmente pentagonal, con cuernos cortos. Escudo con dos surcos medianos, largos y divergentes, en la parte anterior; festones salientes, ampliamente separados entre sí .... A. leucozomum Schulze

Base del capítulo rectangular, con cuernos largos y fuertes. Escudo con dos hoyuelos medianos, cortos y ovales, en la parte anterior; festones no salientes y contiguos ......... ................ A. calcaratum Neumann

48 - Palpos cortos, subcónicos, con una arista saliente que contornea el borde posterior del segundo artículo; primer artículo enormemente ensanchado en la cara ventral. Escudo con manchas esmaltadas pálidas reducidas; festones hacia la cara ventral, ni salientes ni bífidos .... A. nodosum Neumann

Palpos largos, subcilíndricos, sin salientes notorias. Escudo con manchas pálidas esmaltadas, extensas; festones salientes y bifidos en sus ápices, hacia la cara ventral ...... ............... A. incisum Neumann

49 - Coxa III con dos espolones pequeños; coxa II con dos espolones, el interno pequeño. La mayor parte del escudo de color sucio amarillento, vagamente manchado con castaño, sin manchas esmaltadas; punteado abundante, superficial, disperso en las áreas pálidas ........... A. cruciferum Neumann

Coxa III con un espolón ............ 50

50 - Coxa II con dos espolones, el interno muy pequeño $\ldots \ldots \ldots \ldots \ldots \ldots \ldots \ldots \ldots \ldots \ldots$

Coxa II con un espolón ............. 52

51 - Escudo ampliamente ornamentado con manchas y rayas esmaltadas, que simulan hacia la parte anterior el escudo de la hembra; punteado abundante, de tamaño grande y más bien igualmente distribuído ..... ................ A. scutatum Neumann

Escudo pálido castaño, con unas pocas manchas esmaltadas pálidas, cerca del margen, en los festones y delante de ellos; punteado muy escaso, la mayor parte diseminado en la mitad posterior y cerca del margen; casi falta en el área antero-mediana ......... ............ A. deminutivum Neumann

52 - Coxa I con espolones moderadamente largos 0 fuertes . . . . . . . . . . . . . . . 53

Coxa I con espolones pequeños y cortos . . . 55

53 - Coxa I con espolones desiguales, el externo delgado y agudo. Escudo pequeño (2.5 mm.), ampliamente oval, claramente ornamentado, 
con abundante punteado de tamaño mediano y superficial. Festones muy cortos ........ .............. mantiquirense Aragão

Coxa I con espolones anchos, casi iguales. Festones largos .................. 54

54 - Escudo ancho, oval, casi desornamentado, con abundante punteado pequeño. Hipostoma largo, espatulado ....... A. pacue Aragão

Escudo alargado oval, claramente ornamentado con manchas esmaltadas extensas; punteado abundante, grande y profundo, casi uniformemente distribuído. Hipostoma espatulado ................ A. varium Koch

55 - Escudo grande (4.5 mm.), subtriangular, rojizo amarillento, desornamentado, casi liso ; escaso punteado muy fino, localizado la mayor parte en los lados.. A. fulvum Neumann

Escudo pequeño (2 a $3 \mathrm{~mm}$.), oval, o casi tan ancho como largo ................ 56

56 - Escudo vagamente ornamentado, pero las manchas esmaltadas simulan hacia la parte anterior, el escudo de la hembra; festones cortos, casi bien definidos; con una depresión oblicua y curva, que se extiende hacia afuera y hacia adentro, del primer festón de cada lado ............... boulengeri Hirst

Escudo oscuro castaño, desornamentado, con depresiones superficiales, $\sin$ depresiones curvas que se extienden de los primeros festones; festones vagos, con ligeras separaciones; punteado abundante, pequeño $\mathrm{y}$ casi igual $\ldots \ldots \ldots \ldots \ldots \ldots$ A. darwini Hirst

$1-A$. americanum (Linnaeus).-L. H. Dunn (1923, Amer. J. Trop. Med., III, p. 97) anota esta especie en perro y en cerdo de la isla de San Miguel, una de las islas de las Perlas en la bahía de Panamá.

2-A. auricularium (Conil).-Tres hembras fueron colectadas en un armadillo, Dasypus novemcinctus, procedente de Restrepo, Int. del Meta (Orjuela).

3-A. cajennense (Fabricius).-Neumann (1899, Mém. Soc. Zool. France, XII, p. 208) y Robinson (1926, Ticks, IV, p. 53) lo anotan como procedente de Colombia y Panamá. L. H. Dunn (1923, Amer. J1. Trop. Med., III, p. 96) dice que es la especie más importante en Panamá. También refiere que fue la especie común en todas las regiones de Colombia que visitó (1929, Amer. Jl. Trop. Med., IX, p. 507). Se colectaron muchos ejemplares en la región de Villeta, Depto. Cundinamarca, en caballo, cerdo, gato, perro y hombre (J. C. Bequaert y L. Patiño C.) ; en San Vicente de Chucurí, Depto. Santander, en caballo (J. C. Bequaert) ; en Restrepo, Int. del Meta, en caballo, coatí, Nasua socialis, y en hombre (J. C. Bequaert, E. Osorno y J. Boshell).

4-A. crassum Robinson (1926, Ticks, IV, p. 177, fig. 83).-Esta especie fue descrita en ejemplares colectados en una tortuga terrestre procedente del Darién, Rep. Panamá. Siete machos fueron colectados en una tortuga terrestre o "morrocoi" procedente de Restrepo, Int. del Meta (J. Boshell M.). Co- lecté machos en tortugas terrestres, procedentes de Villavicencio, Int. del Meta, (Marzo, 1939).

5-A. deminutivum Neumann (1899, Mém. Soc. Zool. France, XII, p. 221).-Esta especie se basó originalmente en hembras colectadas en una culebra importada de Colombia a París "Jardin des Plantes".

6-Amblyomma dissimile Koch.-Es una garrapata muy común en reptiles y anfibios. Neumann (1899, Mém. Soc. Zool. France, XII, p. 230) la anota como procedente de Colombia y Panamá; Robinson (1926, Ticks, IV, p. 167) la anota procedente de Panamá. De acuerdo con L. H. Dunn (1923, Amer. Jl. Trop. Med., III, p. 97) esta especie predomina en iguana (Iguana tuberculata), en sapo (Bufo marinus), en muchas culebras (Boa imperator; Epicrates cenchria; etc.) en Panamá. Dunn (1929, Amer. Jl. Trop. Med., IX, p. 508) anota esta especie en Colombia en Boa imperator de Barranquilla y en Iguana tuberculata de Sevilla. Fueron colectados ejemplares en Restrepo, Int. Meta, en Bufo marinus (J. C. Bequaert) ; en Bonda, cerca de Santa Marta, Depto. Magdalena, en Boa enydris cookei; y en Aracataca, Depto. Magdalena (J. P. Darlington).

7-A. geayi Neumann (1899, Mém. Soc. Zool. France, XII, p. 223).-Esta garrapata fue originalmente descrita en parte en machos procedentes del Darién, Rep. Panamá. Se colectaron ejemplares en Fort Sherman, C. Z., en Choloepus hoffmanni (R. K. Enders).

8-A. goeldii Neumann.-Robinson (1926, Ticks, IV, p. 244) anota esta especie en un sapo procedente de Condoto, Int. del Chocó.

9 - A. humerale Koch.-L. H. Dunn (1923, Amer. Jl. Trop. Med., III, p. 99) fueron colectados ejemplares en una tortuga en la región del Río Boquerón, Panamá.

10 - A. leucozomum P. Schulze.-Cuatro machos colectados en "oso hormiguero", Tamandua tetradactyla, procedente de Restrepo, Int. del Meta, (J. Boshell M.).

$11-A$. longirostre (Koch).-Un macho se encontró en un puerco-espín, Cercolabes prehensilis, procedente de Muzo, Depto. Boyacá. L. H. Dunn (1923, Amer. Jl. Trop. Med., III, p. 98) lo encontró en Panamá en Coendu rothschildi. Vi un ô colectado en puerco-espín, cercolabes sp., por R. M. Gilmore, (1939).

12 - A. maculatum Koch. - Robinson (1926, Ticks, IV, p. 44) lo anota procedente de Ambalema, Río Magdalena, Depto. Tolima. Un macho fue colectado por Patiño C. en la vereda de Tobia, cerca de Villeta, Depto. Cundinamarca, en la oreja de un caballo.

13 - A. naponense (Packard).-L. H. Dunn (1923, Amer. Jl. Trop. Med., III, p. 99) anota esta especie en un "oso hormiguero", Tamandua tetradactyla chiriquensis, $\mathrm{y}$ en un pecari, Pecari angulatus bangsi, procedentes del Río Boquerón, región de Panamá. A. mantiquirense Aragão es un sinónimo. 
$14-A$. nodosum Neumann.-Tres hembras fueron colectadas en "oso hormiguero", Tamandua tetradactyla, en Restrepo, Int. del Meta (J. Boshell M.). L. H. Dunn (1923, Amer. Jl. Trop. Med., III, p. 98) también lo encontró común en Tamandua tetradactyla chiriquensis en Panamá.

15 - A. oblongoguttatum Koch.-A. darlingi Nuttall (1912, Parasitology, V. p. 50) es un sinónimo y se basó en ejemplares colectados en ciervo y en zopilote, Catharista urubu, en Panamá. De acuerdo con L. H. Dunn (1923, Amer. Jl. Trop. Med., III, p. 97), la especie es comán en Panamá en una variedad de animales domésticos y silvestres y en una ave, Cras panamensis.

16 - A. ovale Koch.-L. H. Dunn (1923, Amer. Jl. Trop. Med., III, p. 100) anota ejemplares colectados en Alhajuela, Panamá, en Tamandua tetradactyla chiriquensis. Estas garrapatas no pertenecen quizá a la especie ovale.

17 - A. pacae Aragão.-Una hembra se encontró en el lado interno de la cadera de una "lapa", Aguti paca, en Restrepo, Int. del Meta (J. Boshell M.).

18-A. parvum Aragão.-L. H. Dunn (1923, Amer, Jl. Trop. Med., III, p. 99) refiere que se colectaron adultos en venado de cola blanca en Panamá; mientras que las larvas y ninfas eran comunes en ratas de las plantaciones de algodón, sigmoden hispidus chiriquensis, cerca de Balboa, Z. del C.

19-A. pecarium Dunn (1933, Parasitology, XXV, p. 356, figs. 3-4). Esta especie fue descrita en ejemplares colectados en pecari, Pecari angulatus bangsi, de Miraflores, Z. del C., Panamá.

20 - A. rotundatum Koch.-Una hembra y varias ninfas se colectaron en un sapo, Bufo marinus, en Restrepo, Int. del Meta (J. C. Bequaert).

21 - A. sabanerae Stoll.-P. Schulze (1937, Zeitschr. f. Parasitenk., IX, p. 692, fig. 3) vio ô y ? de esta especie como procedente de Colombia, sin especificar huésped ni localidad.

22-A. striatum Koch.-Neumann (1899, Mém. Soc. Zool. France, XII, p. 213) lo anota del Darién, Rep. Panamá. Fue colectado en Puerto Boy, Comisaría del Putumayo, por el Dr. L. Patiño C. Ha sido a menudo confundido con $A$. ovale Koch.

23-A. tapirellum Dunn. (1923, Parasitology, $\mathrm{XXV}$, p. 353). Originalmente descrita de Summit, Z. del C., Panamá, proc. de Tapirella bairdii. Machos y hembras también fueron colectados por el extinto Profesor W. M. Wheeler en Barro Colorado, Z. del C.

24-A. varium Koch.--Robinson (1926, Ticks, IV, p. 209) lo anota procedente de un perezoso Bradypus tridactylus, de Condoto, Int. del Chocó; y de Choloepus hoffmanni de Ancón, Z. del C., Panamá. L. H. Dunn (1923, Amer. Jl. Trop. Med., III, p. 98) dice que es comán en Panamá.

\section{HAEMAPHISALIS Koch}

Se conocen dos especies procedentes de Colombia y Panamá.

\section{HEMBRAS}

1 - Escudo más largo que ancho, con punteado tosco, irregular y confluente. Cuernos dorsales muy cortos, menos de un tercio de la longitud de la base del capítulo. Palpos: segundo segmento con el margen dorsal interno bruscamente ensanchado cerca del ápice; tercer segmento sin espina ventral ........ .................

Escudo casi circular o ligeramente más ancho que largo, con punteado fino, igualmente distribuído. Cuernos dorsales largos, más de un tercio de la longitud de la base del capítulo. Palpos: segundo segmento con el margen dorsal interno recto; parte ventral del tercer segmento con un gancho fuerte en forma de espina ........... H. kochi Aragão

\section{MACHOS}

1 - Escudo con punteado denso y tosco, algo confluente en los lados. Cuernos dorsales cortos, menos de un tercio de la longitud de la base del capítulo; pequeño cuerno ventral. Palpos: segundo segmento fuertemente prolongado hacia el margen basal externo, bruscamente ensanchado cerca del ápice en la parte dorsal del margen interno; parte ventral del tercer segmento con una ligera cresta...$\cdots \ldots \ldots \ldots \ldots \ldots$. proxima Aragão

Escudo con punteado muy fino $y$ disperso. Cuernos dorsales largos, más de la mitad de la longitud de la base del capítulo; sin cuernos ventrales. Palpos: parte basal del segundo segmento débilmente saliente, con el margen dorsal interno recto; parte ventral del tercer segmento con un espolón retrógrado muy largo y agudo ............. .................. kochi Aragão

1-Haemaphysalis proxima Aragão.-Machos, hembras y ninfas fueron colectados en un conejo silvestre procedente de Muzo, Depto. Boyacá, por el Dr. M. Roca García. Una ninfa fue colectada también en Muzo, en huésped humano. He visto machos, hembras y ninfas colectados en un conejo silvestre procedente de Casa Blanca, Girardot, Cundinamarca, por A. Gast G. (1940). Aragão describió originalmente esta especie como una variedad de Norte América, H. leporis-palustris (Pakard), pero la comparación de ambas especies demuestra la existencia de diferencias estructurales que parecen de valor específico. L. H. Dunn (1923, Amer. Jl. Trop. Med., III, p. 96) anota H. leporis-palustris como procedente de un conejo doméstico de Panamá (posiblemente importado de Estados Unidos) y también como procedente de un Dasyprocta punctata isthmica silvestre, de Matachin, Z. del C. El último es más probable que haya sido $H$. proxima.

2-Haemaphysalis kochi Aragão.-L. H. Dunn (1923, Amer. J. Trop. Med., III, p. 96) anota esta especie, procedente de venado de cola blanca, Odocoileus chiriquensis, de Frijoles, Panamá. 


\section{BOOPHILUS Curtice}

Se han anotado tres especies procedentes de Colombia y Panamá.

\section{HEMBRAS}

1 - Espinas de la coxa I muy anchas y contiguas. Coxas II y III sin lóbulo. Ojos convejos y algo salientes del margen extremo del escudo ................. annulatus (Say) Espinas o lóbulos de la coxa I separados por una ranura ancha y profunda. Coxas II y III ensanchadas o lobuladas en la parte baja del margen externo. Ojos planos, no salientes del margen del escudo ......... 2

2 - Ojos circulares, colocados cerca del margen del escudo .......... B. cyclops Minning Ojos ovalados, alejados del margen del escudo ........... microplus (Canestrini)

\section{MACHOS}

1 - Cuerpo sin protuberancia mediana caudal. Espina externa de la coxa I delgada, en forma de dedo ............. B. annulatus (Say)

Cuerpo con una protuberancia mediana caudal. Espina externa de la coxa I ampliamente triangular $\ldots \ldots \ldots \ldots \ldots \ldots \ldots \ldots .2$
2 - Espina externa triangular de la coxa I roma ......... microplus (Canestrini)

Espina externa triangular de la coxa I aguda ................. cyclops Minning

1 - De acuerdo con W. Minning (1934, Zeitschr. f. Parasitenk., VII, p. 35), la garrapata común del ganado en Colombia y Panamá es Boophilus (Uroboophilus) microplus (Canestrini). El vio ejemplares procedentes de Cartagena. Esta es probablemente la garrapata anotada por L. H. Dunn (1923, Amer. Jl. Trop. Med., III, p. 95 y 1920, Op. cit., IX, p. 507), con el nombre de Margaropus annulatus australis, de Panamá (prácticamente en ganado de cualquier localidad) y Colombia (Bucaramanga; Barranquilla; y a lo largo del Río Magdalena). $B$. microplus, encontrado en ganado en Pto. Boy, Int. Putumayo; Río Igara-Paraná (La Enea), Int. Amazonas; y la Unión, cerca a Restrepo, Int. Meta.

2 - L. H. Dunn (1923, Amer. Jl. Trop. Med., III, p. 95) también menciona la existencia de Boophilus annulatus (Say) en Panamá, pero probablemente tan sólo en ganado recientemente importado de Estados Unidos.

3-Boophilus (Uroboophilus) cyclops Minning es una especie de Centro América muy cercana a $B$. microplus. Minning incluye a Panamá en la distribución geográfica. Que cyclops sea actualmente distinto de microplus, está por discutir. 